\title{
ALGAS DE LAS LAGUNAS DE ALTA MONTAÑA DE SIERRA NEVADA (GRANADA, ESPAÑA)
}

\author{
Pedro M. SANCHEZ-CASTILLO
}

RESUMEN: Se estudia la composición específica del fitoplancton de 16 lagunas de alta montaña de Sierra Nevada. Las lagunas estudiadas se encuentran situadas sobre sustrato siliceo y con una altitud comprendida entre los 2.500 y los $3.100 \mathrm{~m}$. Presentan un período libre de hielo que oscila entre 5 y 6 meses, normalmente de juinio a octubre. De los 149 taxones catalogados, 21 pertenecen a las Cyanophyceae, 2 a las Dinophyceae, 8 a las Euglenophyceae, 5 las Chrysophyceae, 51 a las Diatomophyceae, 28 a las Chlorophyceae y 34 a las Zygophyceae; 11 de los cuales no habían sido citados con anterioridad para la ficoflora española.

Por su especial interés biogeográfico son de destacar Eunotia pseudopectinalis, $E$. sudetica, Netrium oblongum var. curvatum y Actinotaenium adelochondrum var. kriegeri, todos ellos de distribución ártico-alpina, mientras que Nostoc kihlmani, Meridion circulare, Cymbella minuta, Pediastrum boryanum, Cosmarium garrolense y Staurastrum hirsutum son los taxones que presentan una más amplia distribución en el conjunto de las lagunas estudiadas.

Se propone una combinación nueva: Eunotia pseudopectinalis Hustedt var. vasconiensis (Manguin) Sánchez Castillo.

RESUME: Dans ce travail on étudie la composition spécifique du phytoplancton de 16 lacs de haute montagne de Sierra Nevada. Les lacs étudiés sont-ils situés sur le sol silicieux et à une altitude qui est entre 2.500 et $3.100 \mathrm{~m}$. Elles presentent un période livre du gel qui varie entre 5 et 6 mois, normalement entre juin et octobre. Des 149 taxons cités, 21 appartiennent à Cyanophyceae, 2 à Dinophyceae, 8 à Euglenophyceae, 5 à Chrysophyceae, 51 à Diatomophyceae, 28 à Chlorophyceae et 34 à Zygophyceae; 11 d'entre eux n'avaient pas été cités auparavant pour la phycoflore espagnole.

Pour leur intérêt biogeographique on detaché: Eunotia pseudopectinalis, E. sudetica, Netrium oblongum var. curvatum et Actinotaenium adelochondrum var. kriegeri qui ont distribution artic-alpine; tandis que Nostoc kihlmani, Meridion circulare, Cymbella minuta, Pediastrum boryanum, Cosmarium garrolense et Staurastrum hirsutum sont les spèces ayant une distribution plus ample dans l'ensemble des lacs étudiés.

La combinaison nouvelle Eunotia pseudopectinalis Hustedt var. vasconiensis (Manguin) Sánchez Castillo est proposé.

Palabras clave: Algas de agua dulce, fitoplancton, Sierra Nevada, España.

Key words: Fresh-water algae, fitoplancton, Sierra Nevada, Spain.

\section{INTRODUCCION}

Las algas de los sistemas montañosos españoles han sido objeto de atención de pocos autores, entre los que podemos 
señalar a Bachmann (1913), Belloc (1892), Caballero (1944, 1950), González Guerrero (1927, 1929a, 1929b, 1942, 1965), Margalef (1948,1949, 1950,1953,1956), Margalef \& al. (1975), Montiel Frades (1947), Vilaseca (1978) y willen (1960); escasos trabajos teniendo en cuenta que España es uno de los paises más montañosos de Europa.

Dentro de la Península Ibérica es Sierra Nevada el macizo montañoso que alcanza una mayor altitud, siendo paradójicamente uno de los que menor atención ha recibido en sus estudios ficológicos, ya que son bastante recientes todos los realizados. González Guerrero (1975, 1976) describe de forma muy general los "ficótopos" de uno de sus principales valles (Siete Lagunas), Martínez (1975, 1977, 1980, 1981) estudia 1a ecologia del fitoplancton de una de sus lagunas (La Caldera) y Sánchez Castillo (1984) describe las comunidades reófilas de algunos de sus ríos. Más recientemente Sánchez Castillo (1987a, 1987b) da a conocer un nuevo taxon: Chromulina nevadensis, así como la descripción del ciclo biológico de Korshikoviella gracilipes.

Las lagunas estudiadas se encuentran situadas sobre sustrato siliceo y con una altitud comprendida entre 1os 2.500 y los $3.100 \mathrm{~m}$. Presentan un período libre de hielo que oscila entre los 5 y 6 meses, normalmente de junio a octubre. En general se pueden definir como lagunas poco profundas ( 0.5 a 12 m) y de reducido volumen, cuya zona litoral se encuentra desprovista de vegetación, o a lo sumo presenta un reducido cinturón de vegetación acuática constituida exclusivamente por briófitos, siendo Drepanocladus exannulatus 1 a especie más abundante.

\section{MATERIAL Y METODOS}

El material estudiado procede de muestras planctónicas obtenidas de la zona litoral de 16 lagunas, en 10 de las cuales las muestras se tomaron de forma periódica a lo largo de un ciclo libre de hielo (Virgen Superior, Virgen Media, Yeguas, Aguas Verdes, Río Seco Superior, Río Seco, Río Seco Inferior, Caldera, Gemela y Majano), mientras que en las 6 restantes (San Juan, Cuarta, Hondera, Quinta, Veleta y Monachil) se obtuvieron sin ningún tipo de periodicidad.

Se han utilizado dos métodos para la toma de muestras, uno de ellos fue el filtrado con red de Nytal de $10 \mu \mathrm{m}$ de diámetro de poro, fijándolas con formol, y el otro consistió en sedimentar muestras de $100 \mathrm{ml}$ previamente fijadas con lugol.

RESULTADOS

CLASE CYANOPHYCEAE

Familia Chroococcaceae

Aphanothece microscopica Näg .

Células de 6-7 x 3,5-4,5 $\mu \mathrm{m}$; formando agrupaciones más 0 irregulares de 50-60 $\mu \mathrm{m}$ de diámetro. 
Chroococcus minor (Kütz.) Näg.

Fig. 1, B

Células sin vaina de 2,5-3,5 $\mu \mathrm{m}$, con ella de 4-6 $\mu \mathrm{m}$. Agrupaciones de dos células.

Chroococcus tenax (Kirchn.) Hieron

Fig. 1, C

Grosor de las células: sin vaina de 29-36 $\mu \mathrm{m}$, con ella de 45-52 $\mu \mathrm{m}$; en agrupaciones de 2 ó 4 indivíduos. Vaina de color amarillo-verdoso.

Si bien las dimensiones celulares son mayores que las dadas en las descripciones (Geitler, 1932; Skuja, 1964) entre otros, la morfología y coloración de la vaina nos hacen incluir nuestros ejemplares en este taxon.

Chroococcus turgidus (Kütz.) Näg.

Fig. 1, D

Grosor de las células: sin vaina de 12-14 $\mu \mathrm{m}$, con ella de 20-28 $\mu \mathrm{m}$; vaina muy poco estratificada.

Cyanarcus sp.

Fig. 1, E

Células arqueadas en mayor o menor grado, desde semicirculares hasta ligeramente curvadas, con márgenes paralelos en la zona central y convergentes hacia los extremos. Cromatoplasma homogéneo, en la mayoría de los casos con dos gruesos gránulos en ambos extremos celulares. Dimensiones: $1-1,5$ x 5-6 $\mu \mathrm{m}$.

Desde que Martínez (1975) inicia el estudio limnológico de la laguna de La Caldera, este taxon se incluyó bajo la denominación específica de Cyanarcus hamiformis Pascher. Dos son los caracteres morfológicos, que a nuestro juicio, separan el taxon de Sierra Nevada de C. hamiformis. Por una parte sus mayores dimensiones, ya que Pascher (1914) da unas medidas de 3-4 $\mathrm{x}$ 0,5-0,75 $\mu \mathrm{m}$, además de una morfología relativamente diferenciada, no claramente observable en las ilustraciones de Geitler (1932), pero sí en la descripción original; como un tercer carácter diferenciador se puede aludir a la forma de vida, ya que en Sierra Nevada siempre se han observado viviendo de forma aislada, mientras que $C$. hamiformis constituye pequeños tricomas, carácter que llevó a Bourrelly (1970) a incluir este taxon en el género Gomontiella Teodoresco.

Eucapsis alpina Clements et Schanz

Fig. 1, F

Células de 4-5 $\mu \mathrm{m}$ de diámetro, presentándose normalmente en agrupaciones de 8, las cuales ofrecen unas dimensiones de 25-27 x 18-20 $\mu \mathrm{m}$. Considerando las medidas dadas por Prescott \& Vinyard (1965) para la var. major Prescott, y las de la var. minor Skuja, las encontradas en las poblaciones de Sierra Nevada entran dentro del rango de variación de la especie.

Gomphosphaeria lacustris Chodat

Fig. 1, G Células de 2-4 x 1-2 $\mu \mathrm{m}$, formando agrupaciones de hasta $30 \mu \mathrm{m}$ de diámetro.

Merismopedia punctata Meyen

Fig. 1, H-I Células de 3-4 $\mu \mathrm{m}$ de grosor; agrupaciones de 16 células: 25-30 x 18-20 $\mu \mathrm{m}$. En ocasiones se han observado células que parecian contener vacuolas de gas, tal como se observa en la figura 1 , I.

Microcystis holsatica Lemm.

Fig. 1, J

Células muy pequeñas, hasta $1 \mu \mathrm{m}$ de diámetro. Agrupaciones de forma más o menos irregular y con perforaciones.

Microcystis marginata (Menegh.) Kütz.

Fig. 1, K

Células de 4-5 $\mu \mathrm{m}$ de diámetro, presentando vacuolas de gas. Diámetro de la agrupación entre 20 y $50 \mu \mathrm{m}$. 
Synechococcus maior Schroeter

Fig. 1, L

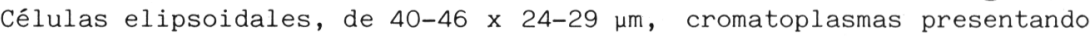
gran cantidad de inclusiones.

Synechococcus sp.

Fig. 1, M

Células de 14-19 x 2,5-3,5 $\mu \mathrm{m}$, viviendo siempre de forma simbionte en el interior del rizópodo Paulinella chromatophora. Si bien pensamos que morfológicamente su inclusión en este género es correcta, actualmente existe la tendencia de considerar a las cianofíceas de vida simbionte como pertenecientes a géneros independientes, debido a las transformaciones a nivel estructural que este tipo de vida les ocasiona.

\section{Familia Stigonemataceae}

Stigonema ocellatum Thuret

Filamentos de 26-38 $\mu \mathrm{m}$, tricoma de 12-24 $\mu \mathrm{m}$.

Fig. 1, N

\section{Familia Nostocaceae}

Anabaena cylindrica Lemm.

Fig. 1, 0

Células vegetativas cuadradas, oscilando entre 2,5-4,5 $\mu \mathrm{m}$ de grosor. Heterocistos de 4-5 x 7-8 $\mathrm{m}$. Acinetos cilíndricos, de 20-30 x 5-8 $\mu \mathrm{m}$.

Anabaena lapponica Borge

Fig. 1, P

Células vegetativas redondeadas, de 7-7,5 $\mu \mathrm{m}$ de diámetro. Heterocistos muy semejantes, hasta $8 \mu \mathrm{m}$ de diámetro. Acinetos cilíndricos, de pared muy gruesa: $35-40 \times 10-15 \mu \mathrm{m}$.

Si bien este taxon presenta una distribución preferentemente nórdica, Hirano (1983) también lo encuentra en hábitats de elevada altitud (3000 m).

Nostoc kihlmani Lemm.

Fig. 1, Q-R

Células vegetativas redondeadas, de 3-5 $\mu \mathrm{m}$ de diámetro, con vacuolas de gas. Heterocistos de 6-8 x 4-7 $\mu \mathrm{m}$. Se han observado agrupaciones de hasta $200 \mu \mathrm{m}$ de longitud.

\section{Familia 0scillatoriaceae}

Oscillatoria agardhii Gom.

Fig. 1, S-T

Células cuadradas, en ocasiones un poco más largas que anchas, con un grosor que oscila entre $3-4,5 \mu \mathrm{m}$. Célula apical un poco adelgazada en su extremo, a veces se observa una pequeña caliptra.

Oscillatoria amoena Gom.

Fig. 1, V

Células cuadradas o más largas que anchas, de 3,5-6 x 4-5 $\mu \mathrm{m}$. Tricoma atenuado en una caliptra más o menos triangular.

Oscillatoria curviceps Ag.

Fig. 1, U

Tricomas gruesos, de 8-10 $\mu \mathrm{m}$ de grosor, células muy cortas, de 1-3 $\mu \mathrm{m}$ de longitud.

Oscillatoria planctonica Woloszynska

Fig. 1, X

Células desde cuadradas hasta más largas que anchas, oscilando sus dimensiones entre $1,5-2 \times 2-3(-6) \mu \mathrm{m}$. 

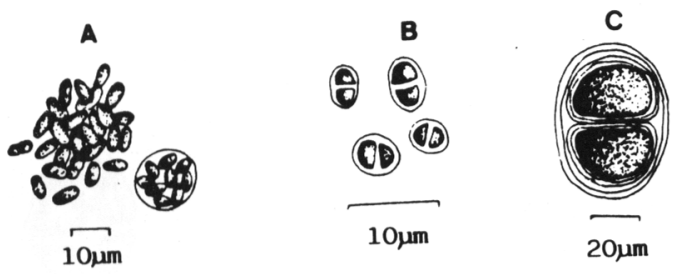

D
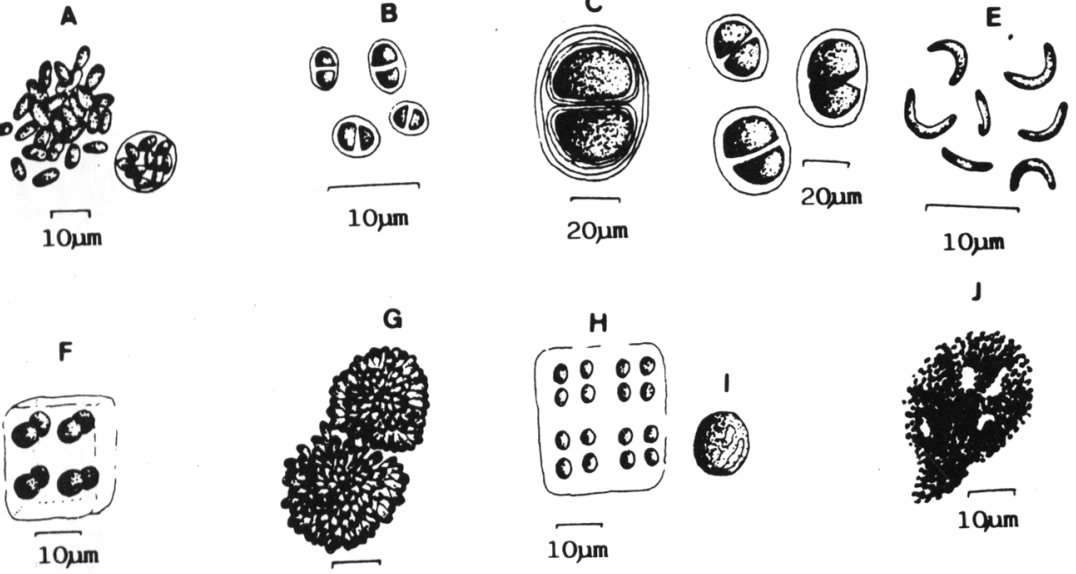

G
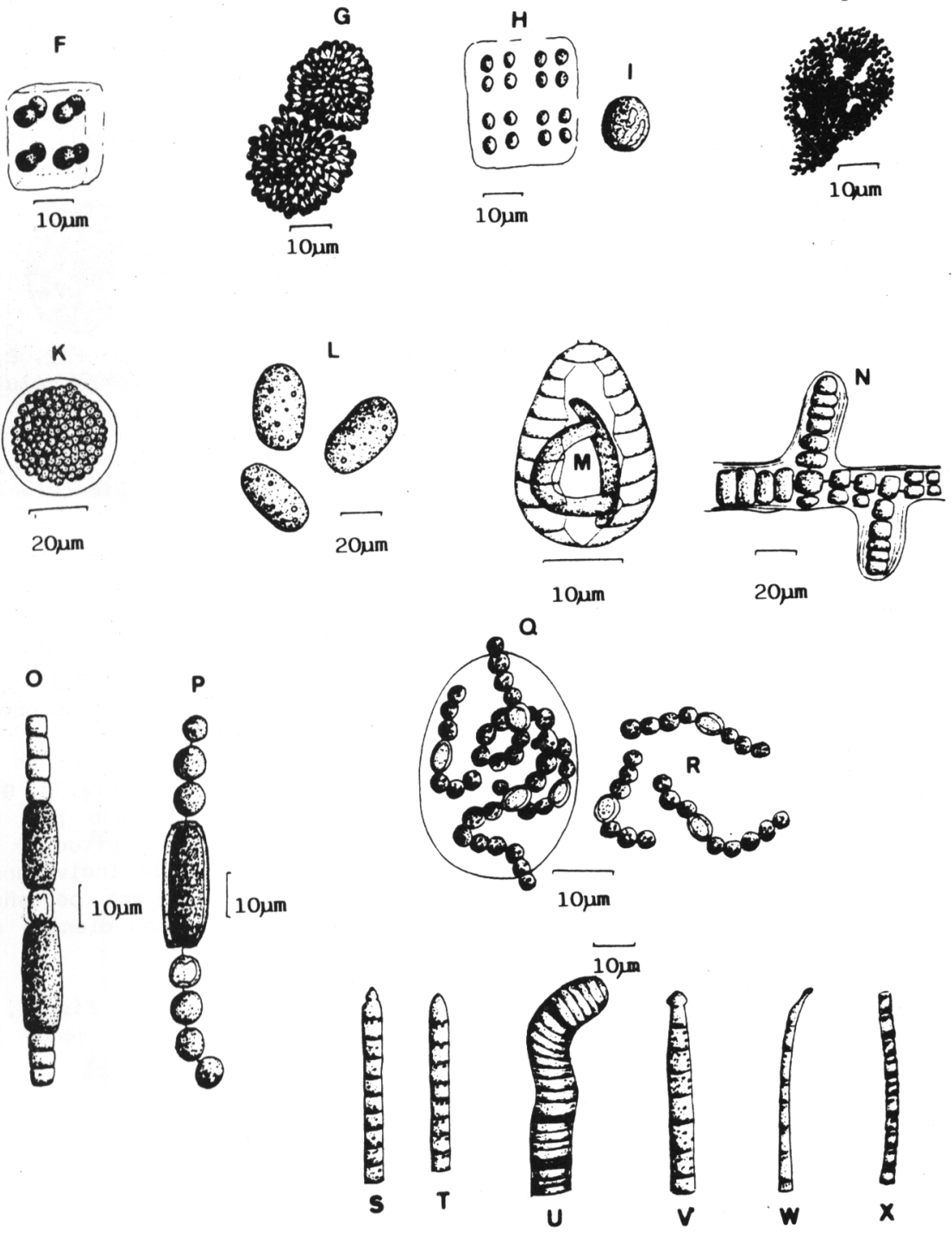

rigura 1.- A, Aphanothece microscopica; B, Chroococcus minor; C, Chroococcus tenax; D, Chroococcus turgidus; E, Cyanarcus sp.; F, Eucapsis alpina; G, Gomphosphaeria lacustris; H-I, Merismopedia punctata; J, Microscystis holsatica; K, Microcystis marginata; L, Synechococcus maior; M, Synechococcus sp.; N, Stigonema ocellatum; 0, Anabaena lapponica; Q, Nostoc kihlmani; S-T, Oscillatoria agardhii; U, Oscillatoria curviceps; V, Oscillatoria amoena; W, 0scillatoria splendida; $X$, 0scillatoria planctonica. 
Oscillatoria splendida Grev.

Fig. 1, W de grosor.

Células de 2-3(-4) veces más largas que anchas, midiendo de 2-2,5 $\mu \mathrm{m}$

\section{CLASE DINOPHYCEAE}

Familia Peridiniaceae

Peridinium umbonatum Stein

Células ovales, de 25-29 x 20-24 $\mu \mathrm{m}$.

Fig. 2, A

Peridinium willei Huitf. - Kaas

Fig. 2, B

Especie de gran tamaño y característica ornamentación areolada de sus placas. Dimensiones: 50-61 x 50-55 $\mu \mathrm{m}$.

\section{CLASE EUGLENOPHYCEAE}

Familia Euglenaceae

Euglena spirogyra Ehr.

Fig. 2, c

Células fuertemente estriadas, flagelo mucho más corto que la célula:

7-8 $\mu \mathrm{m}$. Dimensiones: 90-110 x 9-14 $\mu \mathrm{m}$.

Phacus acuminatus Stokes

Fig. 2, D

Células presentando su máxima anchura en la zona central Dimensiones: $31-33 \times 23-25 \mu \mathrm{m}$.

Phacus lemmermannii (Swir.) Skvortzow

Fig. 2, E

Células fuertemente curvadas en visión lateral. Dimensiones: 45-47 $\mathrm{x}$ 36-39 $\mu \mathrm{m}$.

Trachelomonas abrupta Swir. var. minor Defl.

Fig. 2, F

Células ovoides con lórica amarilla y finamente punteada. Dimensiones: $18-22 \times 10-14 \mu \mathrm{m}$.

Trachelomonas acanthostoma Stokes em. Defl.

Fig. 2, G-H

Lórica esférica, de color ocráceo. Poro flagelar rodeado por un círculo de pequeñas espinas ( 7 u 8). Flagelo de 1,5 veces la longitud de la lórica. Dentro de esta especie se han observado dos tipos de individuos, unos totalmente lisos (Fig. 2, G) y otros ornamentados con pequeñas protuberancias longitudinales y con estrías radiales entre los dientes de la abertura flagelar (Fig. 2, H).

Trachelomonas hispida (Perty) Stein em. Defl.

Fig. 2, I Lórica elipsoidal, de 23-35 x 15-20 $\mu \mathrm{m}$. Flagelo de 1,5 a 2 veces la longitud de la lórica.

Trachelomonas lacustris Drez.

Fig. 2, J Lórica cilíndrica, muy finamente punteada, de 25-27 x 12-14 $\mu \mathrm{m}$.

Trachelomonas verrucosa Stokes var. macrotuberculata Grandori Fig. 2, K-L Lórica ligeramente más larga que ancha, ornamentación formada por pequeñas verrugas de más de $1 \mu \mathrm{m}$ de grosor y más o menos circulares. Poro flagelar rodeado por 4 verrugas alargadas. Flagelo de doble longitud que la lórica. Dimensiones: 19-20 x 17-18 $\mu \mathrm{m}$.

Este taxon fue establecido para aquellos individuos de los Alpes italianos que presentaban una ornamentación mucho más patente que el tipo, según Huber-Pestalozzi (1955) en la descripción original no se daban ningún 


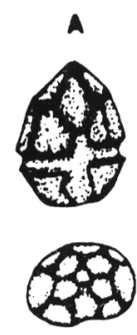

10um

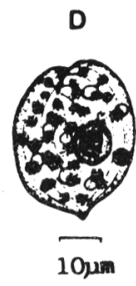

D
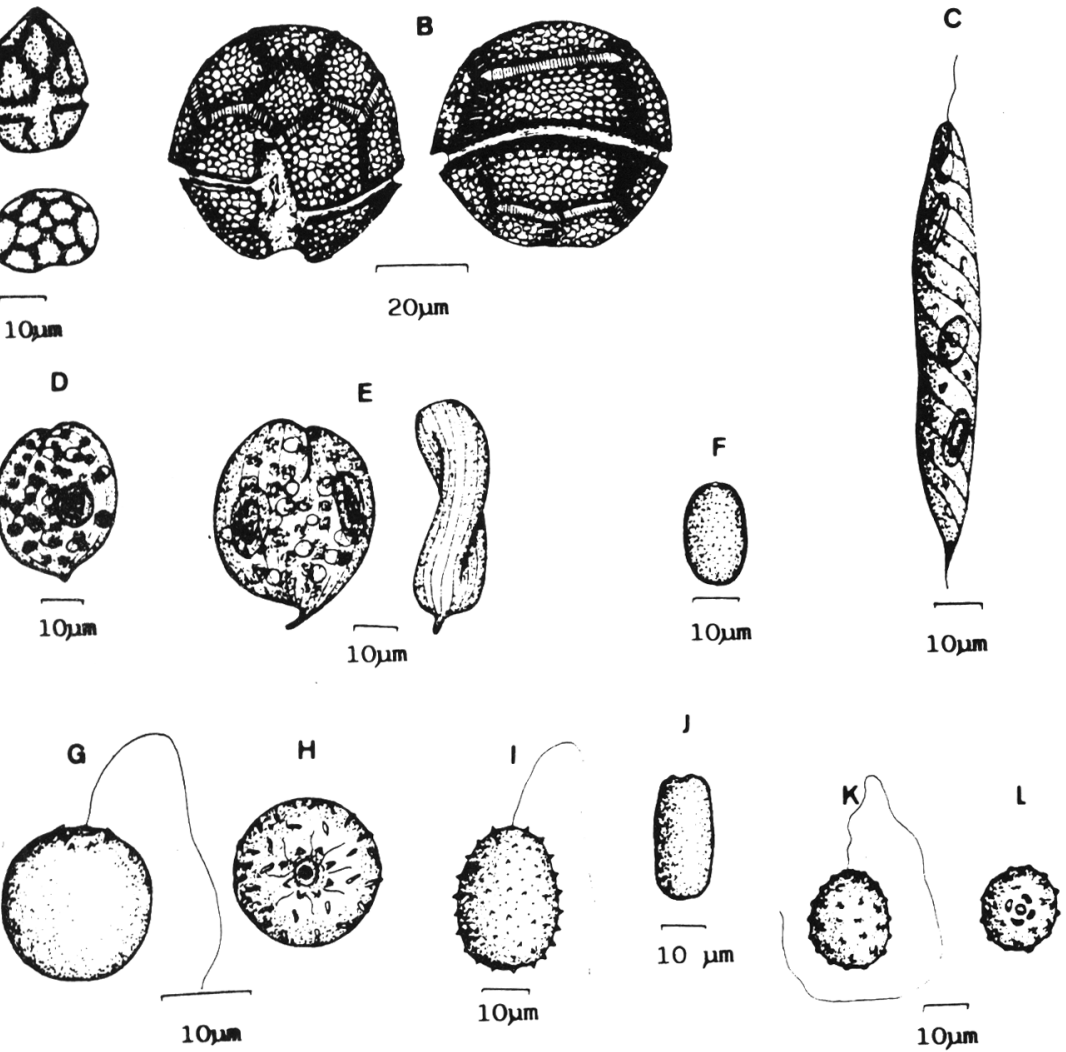

L
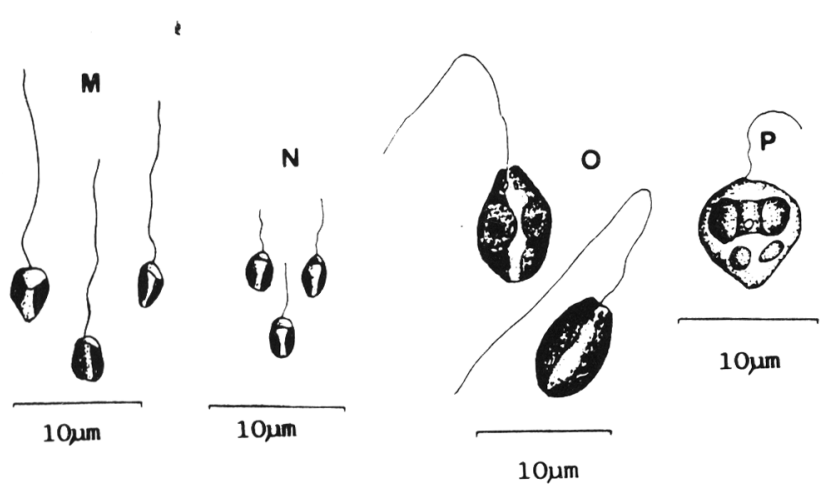

Figura 2.- A, Peridinium umbonatum; B, Peridinium willei; C, Euglena spirogyra; D, Phacus acuminatus; E, Phacus lemmermannii; F, Trachelomonas abrupta var. minor; G-H, Trachelomonas acanthostoma; I, Trachelomonas hispida; J, Trachelomonas lacustris; K-L, Irachelomonas verrucosa var. acrotuberculata; M, Chromulina mikroplankton; $N$, Chromulina minima; 0 , Chromulina nevadensis; P, Chromulina ovaloides; $Q$, Chromulina rosanoffi. 
tipo de dimensiones, pero debido a su característica ornamentación pensamos que debe tratarse de la misma variedad.

\section{CLASE CHRYSOPHYCEAE Familia Chromulinaceae}

Chromulina mikroplankton Pascher

Fig. 2, M

Células muy pequeñas, longitud del flagelo hasta 5 veces superior a la de la célula. Dimensiones: 2,5-4 x 2-2,5 $\mu \mathrm{m}$.

Chromulina minima Doflein Flagelo de longitud ligeramente superior a la de la célula. Dimensiones: $2-3,5 \times 2-2,5 \mu \mathrm{m}$.

Chromulina nevadensis Sánchez Castillo Fig. 2, o célula. Células de 7-8 x 4-5 $\mu \mathrm{m}$, flagelo de 2 a 3 veces más largo que la

Chromulina ovaloides Doflein Fig. 2, P Flagelo de igual longitud que la célula. Dimensiones: 8-10 x 4-7 $\mu \mathrm{m}$.

Chromulina rosanoffi (Woron) Bütschli

Fig. 2, Q Flagelo aproximadamente de igual longitud que la célula. Dimensiones: 8-10 x 4-7 $\mu \mathrm{m}$.

\section{CLASE DIATOMOPHYCEAE Familia Coscinodiscaceae}

Cyclotella meneghiniana Kütz.

Fig. 3, A

Valvas de 15-21 $\mu \mathrm{m}$ de diámetro, presentando de 8-9 estrías marginales de $10 \mu \mathrm{m}$.

\section{Familia Diatomaceae}

Diatoma hiemale (Roth) Heib. var. mesodon (Ehr.) Grun.

Fig. 3, B

Pequeñas valvas elipsoidales, con unas dimensiones comprendidas entre 14-22 x 6-9 $\mu \mathrm{m}$. El número de costillas en $10 \mu \mathrm{m}$ varía entre 2 y 3 .

Fragilaria pinnata Ehr. var. pinnata

Fig. 3, C

Valvas desde linear-elipsoidales hasta casi orbiculares, presentando de 7-8 estrías en $10 \mu \mathrm{m}$. Dimensiones: 3-10 x 2-5 $\mathrm{\mu m}$.

Fragilaria pinnata Ehr. var. trigona (Brun. et Herib.) Hustedt Fig. 3, D Variedad tripolar con pseudorafe en posición lateral en el material estudiado. Diámetro: $10 \mu \mathrm{m}$.

Fragilaria virescens Ralfs

Fig. 3, E Valvas lineares, débilmente atenuadas en los extremos, presentando de 14 a 16 estrías en $10 \mu \mathrm{m}$. Dimensiones: 65-89 × 6-7 $\mu \mathrm{m}$.

Hannaea arcus (Ehr.) Patr.

Fig. 3, F

Syn: Ceratoneis arcus Ehr.

Células arqueadas. Dimensiones: $38-48$ × 6-7 $\mu \mathrm{m}$. 
Meridion circulare (Grev.) Ag.

Fig. 3, G

Valvas de 30-32 x 5-7 $\mu \mathrm{m}$, presentando de 3 a 4 costillas en $10 \mu \mathrm{m}$.

Synedra rumpens Kütz.

Fig. 3, H

Células estrechas, con los ápices capitados, mostrando de 18-20 estrías en $10 \mu \mathrm{m}$. Dimensiones: 48-65 x 2-3,5 $\mu \mathrm{m}$.

Tabellaria flocculosa (Roth) Kütz.

Fig. 3, I

Valvas con estrías muy tenues, de 14 a 18 en $10 \mu \mathrm{m}$. Dimensiones: 20-28 x 8-9 $\mu \mathrm{m}$.

\section{Familia Eunotiaceae}

Eunotia bigibba Kütz.

Fig. 3, J

Valvas con los ápices poco capitados. De 12 a 14 estrías en $10 \mu \mathrm{m}$.

Dimensiones: 28-45 x 9,5-12 $\mu \mathrm{m}$.

Eunotia lunaris (Ehr.) Grun.

Fig. 3, K

Valvas con los extremos atenuados en el margen dorsal, presentando de 14 a 16 estrías en $10 \mu \mathrm{m}$. Dimensiones: 40-60 × 4-5 $\mu \mathrm{m}$.

Eunotia pectinalis (Müll.) Rabh. var. minor (Kütz.) Rabh.

Fig. 3, L Valvas con el margen dorsal plano-convexo y el ventral ligeramente cóncavo, de 10-11 estrías en $10 \mu \mathrm{m}$. Dimensiones: 30-35 x 7-10 $\mathrm{m}$.

Eunotia pseudopectinalis Hustedt

Fig. 3, M-N

Valvas lineares, rectas $O$ indistintamente curvadas, ofreciendo un aspecto sinuoso. Extremos levemente atenuados en el margen dorsal. Rafe prolongada por un canal que atraviesa las estrías, introduciéndose ligeramente hacia el centro de la valva. De 11 a 14 estrías en $10 \mu \mathrm{m}$, cruzadas por una línea hialina en el margen ventral. Dimensiones: 125-194 x 9-11 $\mu \mathrm{m}$.

Manguin (in Allorge \& Manguin, 1941) define un nuevo taxon a nivel varietal: Eunotia flexuosa (Bréb.) Kütz. var. vasconiensis Manguin, sobre material procedente del sur de Francia, el cual morfológicamente se debe incluir en E. pseudopectinalis, ya que coincide con los caracteres distintivos de este taxon, pero sus dimensiones son totalmente diferentes, si las comparamos con las descripciones dadas por Hustedt (1959) y con las ofrecidas en este trabajo, por lo que creemos correcto mantener la variedad establecida para este taxon como E. pseudopectinalis Hustedt var. vasconiensis (Manguin) Sánchez Castillo comb. nov. ( E. flexuosa (Bréb.) Kütz. var. vasconiensis Manguin in Allorge et Manguin. Bull. Soc. Bot. France, 88:175 (1941).).

Eunotia sudetica Müller

Fig. 3, 0 Valvas con el margen ventral casi recto, presentando de 13-15 estrías en $10 \mu \mathrm{m}$. Dimensiones: 20-25 x $5 \mu \mathrm{m}$.

Las medidas para las poblaciones de esta especie en Sierra Nevada, tanto en dimensiones como en número de estrías, coinciden con las dadas por Margalef (1948), Hirano (1972) y Wuthrich (1975), pero no así con las de Hustedt (1959). Wuthrich (loc. cit.) examinó el material estudiado por Hustedt (1959), encontrando las mismas dimensiones que en el estudiado por ella, por lo que las ofrecidas por Hustedt (1959) deben tomarse con cierto espiritu crítico. Teniendo en cuenta los trabajos antes mencionados, sus dimensiones serían: 18-25 x 4-5 $\mu \mathrm{m}$, mientras que el número de estrías comprendido en $10 \mu \mathrm{m}$ sería de 13-15. 
Familia Achnanthaceae

Achnanthes minutissima Kütz.

Fig. 3, P

Valvas linear-lanceoladas, con los ápices engrosados y redondeados. Estrías muy finas. Dimensiones: 13-19 x 3-3,5 $\mu \mathrm{m}$. Por la morfología de los ápices celulares se asemeja a la var. cryptocephala Grun., pero en ésta no se ha descrito área central. La existencia de tal área central, lo aproxima a A. affinis Grun., pero en nuestros ejemplares esta estructura no llega al margen valvar.

Achnanthes lanceolata (Bréb.) Grun.

Fig. 3, Q Valvas lanceoladas, presentando de 14-17 estrías en $10 \mu \mathrm{m}$. Dimensiones: $14-22 \times 5-6 \mu \mathrm{m}$.

Achnanthes linearis (W. Smith) Grun. var. pusilla Grun. Fig. 3, R Valvas lineares, con los extremos redondeados, presentando de 16-19 estrías en $10 \mu \mathrm{m}$. Dimensiones: 14-15 x 3,5-4,5 $\mu \mathrm{m}$.

Cocconeis plancentula Ehr. var. lineata (Ehr.) Cleve De 20-22 estrías en $10 \mu \mathrm{m}$. Dimensiones: 17-27 x 9-19 $\mu \mathrm{m}$.

Fig. 3, S

\section{Familia Naviculaceae}

Anomoeoneis serians (Bréb.) Cleve var. brachysira (Bréb.) Cleve Fig. 3, T Valvas lanceoladas, de 25-30 estrías en $10 \mu \mathrm{m}$. Dimensiones: 20-27 $\mathrm{x}$ 7-8 $\mu \mathrm{m}$.

Caloneis ventricosa (Ehr.) Meist

Fig. 3, U

Valvas con el margen triondulado, estrías poco radiales de 20-25 en 10 $\mu \mathrm{m}$, acortándose en el centro de la valva para dar lugar a un área central circular que alcanza el margen unilateralmente por la desaparición de 1 a 3 estrías. Dimensiones: 45-58 x 10-12 $\mu \mathrm{m}$.

De los taxones establecidos a nivel varietal, es la var. subondulata (Grun.) Patr. la más próxima a las poblaciones de Sierra Nevada, aunque en ésta, el área central alcanza ambos márgenes de la valva y es más regular.

Frustulia rhomboides (Ehr.) De Toni

Fig. 3, V Valvas lanceoladas, con estrías imperceptibles a M.0. Dimensiones: 36-50 x 11-13 $\mu \mathrm{m}$.

Navicula cocconeiformis Greg. ex Grev.

Fig. 3, W Valvas elípticas, estrías claramente radiales, de 25-30 en $10 \mu \mathrm{m}$. Dimensiones: $16-18 \times 8-10 \mu \mathrm{m}$.

Navicula cryptocepahala Kütz.

Fig. 3, X Valvas lanceoladas, presentando de 16-18 estrías en $10 \mu \mathrm{m}$. Dimensiones: $32-35 \times 6-8 \mu \mathrm{m}$.

Navicula laevissima Kütz.

Fig. 3, Y

Valvas de contorno linear, con 17-19 estrías en $10 \mu$ en su zona central. Dimensiones: 21-47 x 6-10 $\mu \mathrm{m}$. El número de estrías en la zona central excede ligeramente a los dados por Hustedt (1961-1966), Patrick \& Reimer (1966) y Germain (1981).

Navicula pseudoscutiformis Hustedt

Fig. 3, Z Valvas casi circulares, presentando de 20-25 estrías en $10 \mu \mathrm{m}$. Dimensiones: 11-13 x 9-10 $\mathrm{m}$. 


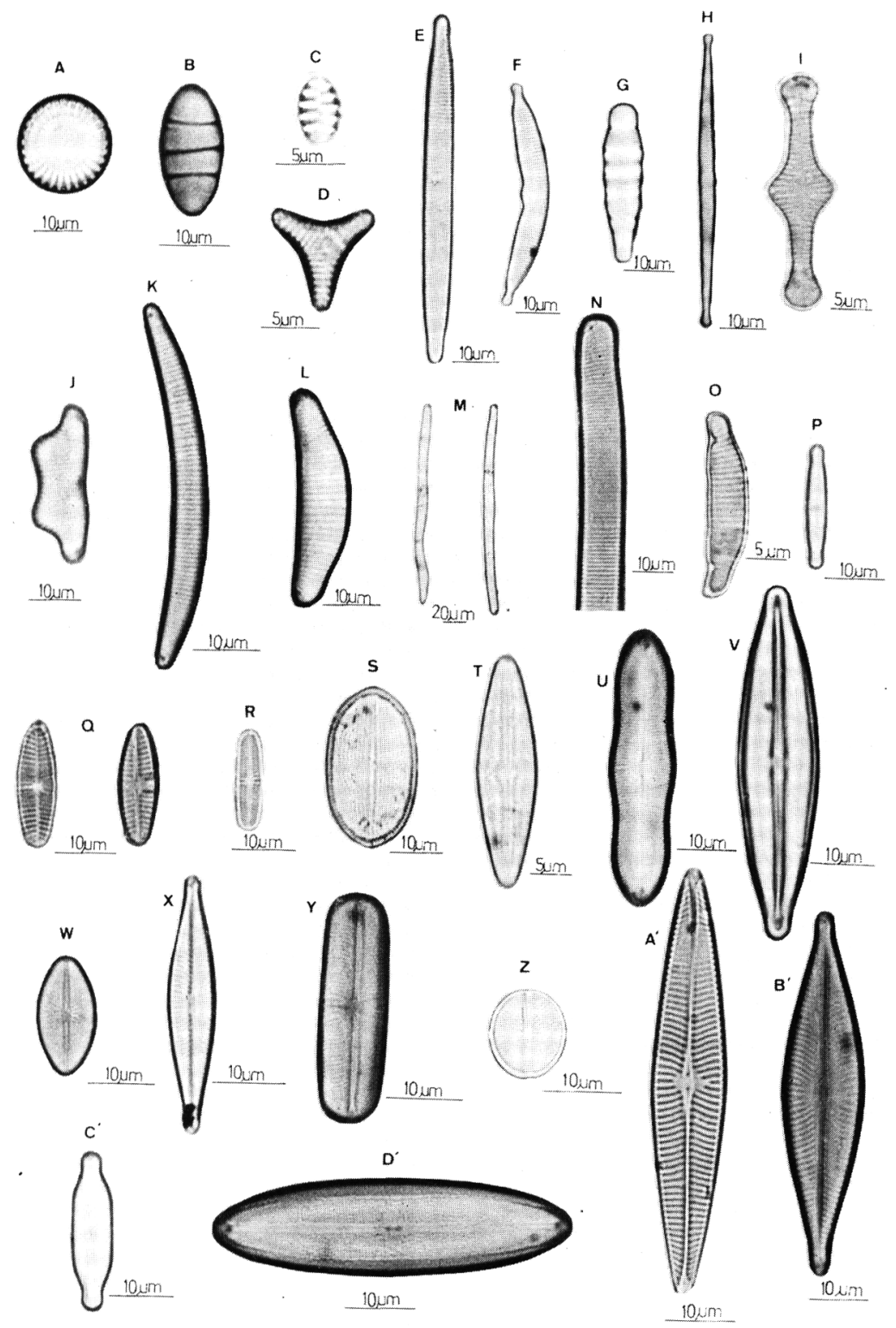

Figura 3.- A, Cyclotella meneghiniana; B, Diatomea hiemale var. mesodon; C, Fragiliaria pinnata var. pinnata; D, Fragiliaria pinnata var. trigona; E, Fragiliaria virescens; F, Hannaea arcus; G, Meridion circulare; H, Synedra rumpens; I, Tabellaria flocculosa; J, Eunotia bigibba; K, Eunotia lunaris; L, Eunotia pectinalis var. minor; M-N, Eunotia pseudopectinalis ; 0, Eunotia sudetica; P. Achnanthes minutissima; Q, Achnanthes lanceolata; $R$, Achnanthes linearis var. pusilla; S, Cocconeis placentula var. lineata; T, Anomoeoneis serians var. brachysira; U, Caloneis ventricosa; V, Frustulia rhomboides; W, Navicula cocconeiformis; X, Navicula cryptocephala; $Y$, Navicula laevissima; $Z$, Navicula pseudoscutiformis; A', Navicula radiosa; B', Navicula rhynchocephala; C', Neidium affine var. longiceps; D', Neidium bisulcatum. 
Navicula radiosa Kütz.

Fig. 3, $A^{\prime}$

Valvas lanceoladas, con un número de estrías que oscila entre 10 y 13 en $10 \mu \mathrm{m}$. Dimensiones: 41-60 x 10-14 $\mu \mathrm{m}$.

Navicula rhynchocephala Kütz.

Valvas lanceoladas, presentando de $11-13$ estrías en 10 m. Dimensiones: $50-55 \times 11-12 \mu \mathrm{m}$.

Neidium affine (Ehr.) Pfitz. var. longiceps (Greg.) Cleve

Valvas ligeramente atenuadas, estrías muy finas, de 26-30 en $10 \mu \mathrm{m}$ y paralelas a lo largo de toda la valva. Dimensiones: 25-35 x 6-7 $\mu \mathrm{m}$. Relación grosor centro/ápice: 2/1.

Taxon muy semejante a N. affine var. amphirhynchus (Ehr.) Cleve, del que se diferencia fundamentalmente en los siguientes caracteres: mayor número de estrías, menor relación grosor centro/ápice y más reducidas dimensiones.

Neidium bisulcatum (Lagerst.) Cleve

Fig. 3, D'

Valvas elípticas, presentando de 25-27 estrías en 10 m. Dimensiones: 45-73 x 11-15 $\mu \mathrm{m}$.

Neidium iridis (Ehr.) Cleve var. ampliatum (Ehr.) Cleve Fig. 4, A Valvas elípticas con los márgenes paralelos en el centro, con aproximadamente 18-20 estrías en $10 \mu \mathrm{m}$. Dimensiones: 60-70 x 17-20 $\mathrm{m}$.

Pinnularia borealis Ehr.

Fig. 4, B

Valvas con gruesas costillas que no sobrepasan de 5 a 7 en $10 \mu \mathrm{m}$. Dimensiones: 29-60 x 9-17 $\mu \mathrm{m}$.

Pinnularia episcopalis Cleve

Fig. 4, C

Valvas muy gruesas, presentando un claro estauro central y patentes nódulos. Estrías centrales radiales, las apicales claramente convergentes; muy gruesas, de 6-8 en $10 \mu \mathrm{m}$. Dimensiones: 110-120 x 34-35 $\mu \mathrm{m}$.

Pinnularia hemiptera (Küt.) Cleve

Fig. 4, D

Valvas con el área axial lanceolada, muy amplia. Presenta de 8-10 estrías en $10 \mu \mathrm{m}$. Dimensiones: 60-65 x 13-14 $\mu \mathrm{m}$.

Pinnularia microstauron (Ehr.) Cleve

De 9-12 estrías en $10 \mu \mathrm{m}$. Dimensiones: 34-67 x 7-12 $\mu \mathrm{m}$.

Fig. 4, E

Pinnularia viridis (Nitzsch) Ehr.

De 12-14 estrías en $10 \mu \mathrm{m}$. Dimensiones: 46-53 x 10-12 $\mu \mathrm{m}$.

Fig. 4, F

Stauroneis anceps Ehr.

Fig. 4, G

Valvas con los ápices reducidos y ligeramente capitados, presentando de 19-20 estrías en $10 \mu \mathrm{m}$. Dimensiones: 32-69 x 9-10 $\mu \mathrm{m}$.

Stauroneis phoenicenteron (Nitzsch) Ehr.

Fig. 4, H

Valvas elíptico-lanceoladas, con 13-18 estrías en $10 \mu \mathrm{m}$. Dimensiones: 98-140 x 19-22 $\mu \mathrm{m}$.

Stauroneis smithii Grün.

Fig. $4, I$

Valvas presentando de 26-30 estrías en $10 \mu \mathrm{m}$. Dimensiones: 15-30 x 4-6 $\mu \mathrm{m}$.

Gomphonema affine Kütz.

Syn.: Gomphonema lanceolatum Ehr.

Valvas lanceoladas, con la parte basal más atenuada que la apical. De 10-12 estrías en $10 \mu \mathrm{m}$. Dimensiones: 24-41 x 5-8 $\mu \mathrm{m}$. 


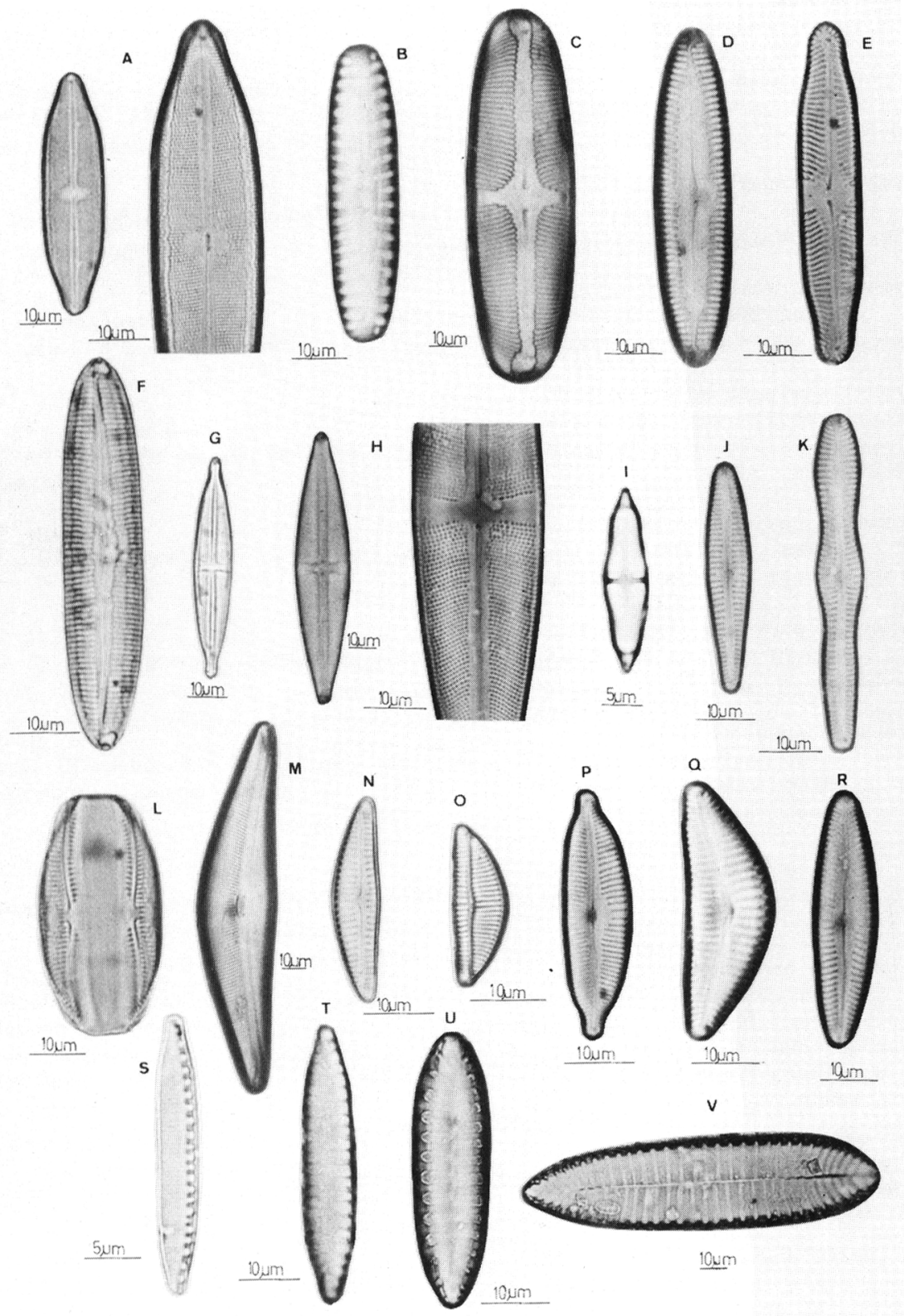

Figura 4.- A, Neidium iridis var. ampliatum; B, Pinnularia borealis; C, Pinnularia episcopalis; D, Pinnularia hemiptera; E, Pinnularia microstauron; F, Pinnularia viridis; G, Stauroneis anceps; H, Stauroneis phoenicenteron; I, Stauroneis smithii; J, Gomphonema affine; K, Gomphonema longiceps; L, Amphora ovalis; M, Cymbella aspera; N, Cymbella gracilis; 0 , Cymbella minuta; P, Cymbella naviculiformis; Q, Cymbella parva; R, Cymbella subaequalis; $S$, Nitzschia hantzschiana; T, Surirella angustata; U, Surirella linearis; V, Surirella tenera. 
Gomphonema longiceps Ehr.

Fig. 4, K Valvas trionduladas, presentando la zona subapical del mismo grosor que la central. De 10-12 estrías en $10 \mu \mathrm{m}$. Dimensiones: $55-60$ x 8-9 $\mu \mathrm{m}$.

Amphora ovalis Kütz.

Fig. 4, L.

Valvas ovales, presentando de 15-20 estrías en $10 \mu \mathrm{m}$. Dimensiones: $38-60 \times 20-25 \mu \mathrm{m}$.

Cymbella aspera (Ehr.) Perag.

Fig. 4, M

Valvas de gran tamaño, estrías claramente punteadas, de 8-9 en $10 \mu \mathrm{m}$. Dimensiones: $140-160 \times 30-35 \mu \mathrm{m}$.

Cymbella gracilis (Rabh.) Cleve

Fig. 4, N

Valvas presentando de 10-14 estrías en $10 \mu \mathrm{m}$, a excepción de los extremos donde se hacen mucho más densas. Dimensiones: 21-34 x 6-7 $\mu \mathrm{m}$.

Cymbella minuta Hilse ex Rabh.

Fig. 4, O

Syn.: Cymbella ventricosa Kütz.

Valvas pequeñas, presentando de 12-16 estrías en $10 \mu \mathrm{m}$. Dimensiones: $13-27 \times 6-8,5 \mu \mathrm{m}$.

Cymbella naviculiformis Aversw. ex Heib.

Fig. 4, P

Valvas con los ápices capitados, de 10-14 estrías en $10 \mu \mathrm{m}$.

Dimensiones: 25-34 x 8,5-10 $\mu \mathrm{m}$.

Cymbella parva ( $W$. Smith) Cleve

Fig. 4, Q

De 8-10 estrías en $10 \mu \mathrm{m}$, fuertemente punteadas. Dimensiones: 32-49 x 10-14 $\mu \mathrm{m}$.

Cymbella subaequalis Grun.

Fig. 4, R

Valvas naviculoideas, con los extremos débilmente atenuados. El número de estrías varía de 9-11 en $10 \mu \mathrm{m}$. Area axial ancha, lanceolada. Dimensiones: $31-37 \times 9-10 \mu \mathrm{m}$.

\section{Familia Nitzschiaceae}

Nitzschia hantzschiana Rabenh.

Fig. 4, S

Valvas lineares, atenuadas en los ápices. De 28-34 estrías y de 8-10 fíbulas en $10 \mu \mathrm{m}$. Dimensiones: 22-35 x 3-4,5 $\mu \mathrm{m}$. Forma pequeñas agrupaciones tabulares. Por el número de estrías se aproxima a $N$. acidoclinata (Lang.) Bert. pero el resto de los caracteres son propios de este taxon.

\section{Familia Surirellaceae}

Surirella angustata Kütz.

Fig. 4, T

Valvas isopolares, presentando de 5-8 costillas en $10 \mu \mathrm{m}$, dejando un fino canal en el centro de la valva. Dimensiones: 27-36 x 7-8 $\mu \mathrm{m}$.

Surirella linearis $W$. Smith

Fig. 4, U

Valvas isopolares, elípticas, costillas en número de $3(-4)$ en $10 \mu \mathrm{m}$. Dimensiones: $34-35 \times 9-10 \mu \mathrm{m}$.

Valvas heteropolares, presentando aproximadamente 30 costillas en 100 $\mu \mathrm{m}$. Dimensiones: 84-120 x 30-35 $\mu \mathrm{m}$. 
CLASE CHLOROPHYCEAE

Familia Chlamydomonadaceae

Chlamydomonas microsphaerella Pascher et Jah.

Fig. 5, A

Células esféricas, con un cloroplasto urceolado presentando un pirenoide basal. Flagelos de doble longitud que la célula. Diámetro de 6-7 $\mu \mathrm{m}$.

Polytoma angustum Pascher

Fig. 5, C Células gutiformes, con el citoplasma ocupado por pequeñas vacuolas y un pirenoide. Dimensiones: 24-26 x 11-13 $\mu \mathrm{m}$.

\section{Familia Volvocaceae}

Pandorina morum (Müller) Bory

Fig. 5, B Normalmente aparece en colonias de 16 células, cuyo diámetro oscila entre 27-41 $\mu \mathrm{m}$.

\section{Familia Tetrasporaceae}

Tetraspora lemmermannii Fott

Fig. 5, D Células de 7-10 $\mu \mathrm{m}$ de diámetro, dispuestas en 3 ó 4 agrupaciones dentro de la envoltura gelatinosa. Pseudoflagelos de 2 a 5 veces el diámetro de la célula. El conjunto de las agrupaciones (incluida la vaina) ofrece un diámetro que varía entre 180-220 $\mu \mathrm{m}$.

\section{Familia Glococystaceae}

Chlamydocapsa planctonica (W. West et G.S. West) Fott

Fig. 5, E-G

Células esféricas que se reúnen formando agrupaciones de forma más o menos piramidal, en ocasiones aparecen aisladas. Agrupaciones de 72-100 $\mu \mathrm{m}$ de diámetro.

\section{Familia Chlorangiellaceae}

Chlorangiella pygmaea (Ehr.) Silva

Fig. 5, H Dimensiones celulares: 12-20 x 5-12 $\mu \mathrm{m}$; pedúnculo de fijación de longitud inferior a la célula. Taxon epizoico sobre cladóceros (Alona).

\section{Familia Chlorococcaceae}


Tetraedron caudatum (Corda) Hansg.

Fig. 5, I

Células pentagonales con un pequeño aguijón sobre cada ángulo, el de mayor longitud puede llegar a medir hasta $3 \mu \mathrm{m}$ de longitud. Dimensiones: 10-12 x 7-12 $\mu \mathrm{m}$.

\section{Familia Oocystaceae}

Ankistrodesmus falcatus (Corda) Ralts

Células fusiformes, formando agrupaciones laxas de morfología estrellada. Dimensiones celulares: 20-60 x 2-3,5 $\mu \mathrm{m}$.

Oocystis lacustris Chod.

Fig. $5, M-N$ 5-7 $\mu \mathrm{m}$.

Células adultas generalmente con 4 cloroplastos. Dimensiones: 10-15 x

Oocystis marssonii Lemm.

Fig. 5, 0

Un solo cloroplasto parietal; células normalmente reunidas en agrupaciones de 4. Dimensiones: 13-18 x 8-9 $\mu \mathrm{m}$.

Oocystis solitaria Wittr.

Engrosamientos polares sólo

Dimensiones: $19-35 \times 14-27 \mu \mathrm{m}$.

Scotiella tuberculata Bourr.

Fig. 5, R-S

Células más o menos ovales, presentando de 7 a 9 costillas longitudinales, onduladas y sinuosas. Dimensiones: 10-15 x 7-12 $\mu \mathrm{m}$.

\section{Familia Radiococcaceae}

Eutetramorus fottii (Hindak) Kom.

Fig. 5, T

Células esféricas, de 9-12 um de diámetro, agrupándose en número de 4, 8 ó 16. Diámetro de la agrupación: 60-110 $\mu \mathrm{m}$.

\section{Familia Scenedesmaceae}

Actinastrum hantzschii Langer

Células de 12-15 x 2-3 $\mu \mathrm{m}$. Relación longitud-grosor: 5-7.

Fig. 5, U

Coelastrum microporum Näg.

Fig. 6, A

Diámetro de las células: 9-11 $\mu \mathrm{m}$; del cenobio de 8 células: 23-28 $\mu \mathrm{m}$; del cenobio de 16 células: 50-55 $\mu \mathrm{m}$.

Scenedesmus corallinus Chod.

Fig. 6, B

Dimensiones de las células: 8-13 x 2-5 $\mu \mathrm{m}$; del cenobio: 8-13 x 16-20 $\mu \mathrm{m}$.

Scenedesmus pseudohystrix Masj.

Fig. 6, G

Syn.: Scenedesmus denticulatus Langer

Dimensiones de las células internas: 15-16 x 5-6 $\mu \mathrm{m}$; de las externas: 13-14 x 5-6 $\mu \mathrm{m}$; del cenobio: 15-16 x 20-25 $\mu \mathrm{m}$. 

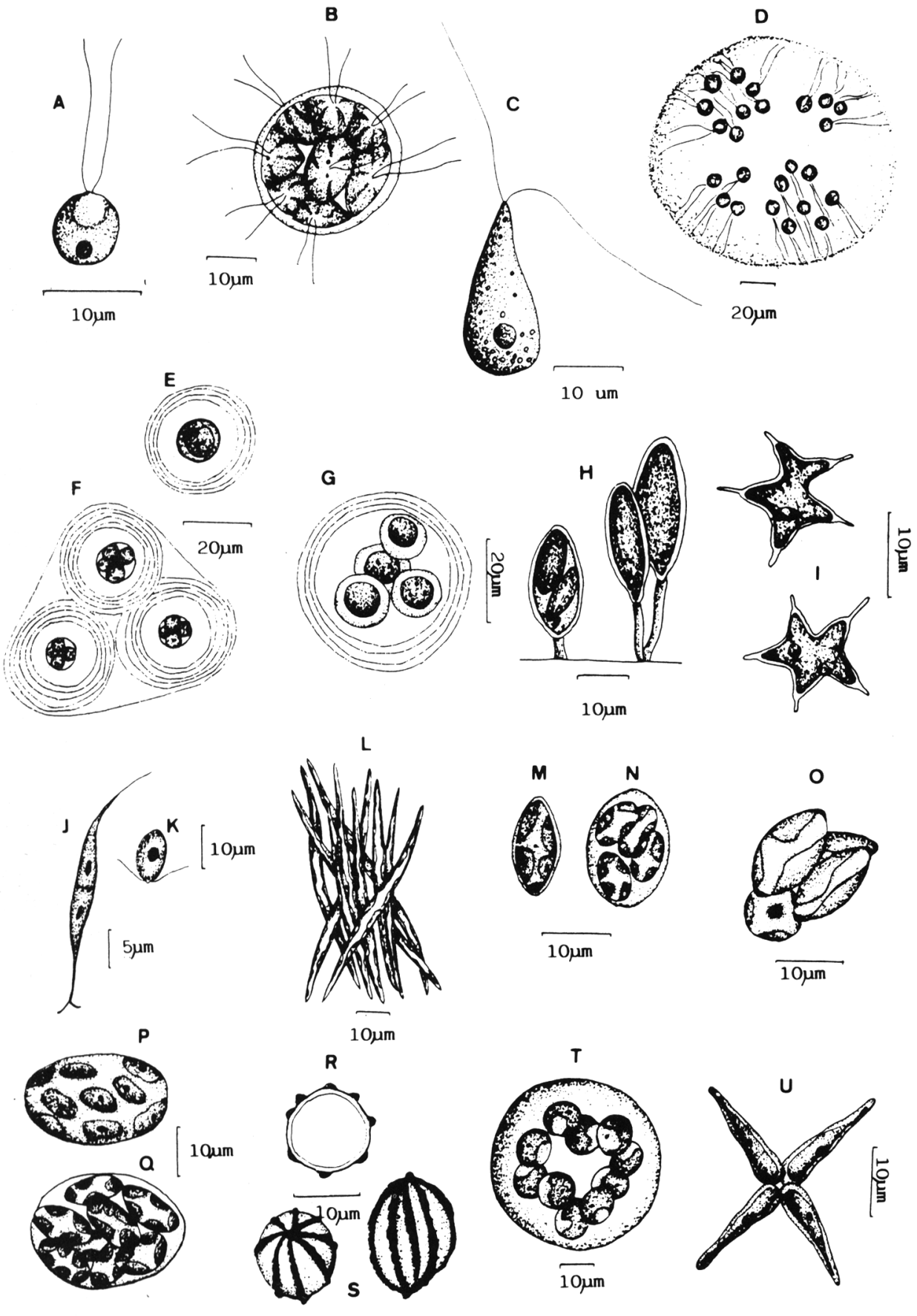

Figura 5.- A, Chlamydomonas microsphaerella; B, Pandorina morum; C, Polytoma angustum; D, Tetraspora lemmermannii; E-G, Chlamydocapsa planctonica (distintos estadios); H, Chlorangiella pygmaea; I, Tetraedron caudatum; J-K, Korshikoviella gracilipes (J, organismo ankiroide, K, zoospora); L, Ankistrodesmus falcatus; M-N, 0ocystis lacustris; 0, 0ocystis márssonii; P-Q, Oocystis solitaria; R-S, Scotiella tuberculata; T, Eutetramorus fottii; U, Actinastrum hantzschii. 
Scenedesmus quadrispina Chod.

Fig. 6, D Syn.: Scenedesmus quadricauda (Turpin) Bréb. var. quadrispina (Chod.) G. Smith

Dimensiones celulares: 22-27 x 6-7 $\mu \mathrm{m}$; longitud de las espinas: 7-10 $\mu \mathrm{m}$.

\section{Familia Hydrodictyaceae}

Pediastrum boryanum (Turpin) Menegh.

Fig. 6, E Células externas de 8-17 x 5-15 $\mu \mathrm{m}$. Ornamentación desde casi imperceptible hasta gruesamente granular. Diámetro de los cenobios: 50-100 $\mu \mathrm{m}$.

Pediastrum boryanum (Turpin) Menegh. var. cornutum (Racib.) Sulek Fig. 6, F Células externas de 7-17 x 6-14 $\mu \mathrm{m}$. Cenobio presentando perforaciones constantemente. Ornamentación siempre patente, generalmente granulosa, aunque en ocasiones con una clara tendencia rugulosa, sobre todo en las células externas. Diámetro de los cenobios: 30-70 $\mu \mathrm{m}$.

Pediastrum braunii Wart.

Fig. 6, G Dimensiones de las células: 8-13 x 14-17 $\mu \mathrm{m}$. El diámetro de los cenobios varía entre 23-40 $\mu \mathrm{m}$.

Pediastrum tetras (Ehr.) Ralfs

Fig. 6, H Unicamente se han observado cenobios de 4 células. Células de 7-8 $\mu \mathrm{m}$. Cenobios de 17-19 $\mu$ m de diámetro.

\section{Familia Ulotrichaceae}

Geminella ordinata (W. West \& G.S. West) Heering

Fig. 6, I Dimensiones celulares: 5-7 x 4-5 $\mu \mathrm{m}$. Filamentos de 10-11 $\mu \mathrm{m}$ de grosor.

Ulothrix subconstricta G.S. West

Fig. 6, J Dimensiones celulares: 15-26 × 3-5 $\mu \mathrm{m}$; no se ha observado nunca la presencia de vaina mucosa.

Ulothrix tenerrima (Kütz.) Kütz.

Fig. 6, K Dimensiones celulares: $3-6 \times 6-8 \mu \mathrm{m}$.

\section{Familia Oedogoniaceae}

Oedogonium rothii (Le Clerc) Prings. Fig. 6, L Células de 24-36 x 5-10 $\mu \mathrm{m}$; nanandro urceolado; 12-15 x 8-14 $\mu \mathrm{m}$; cigoto elipsoidal de 20-24 x 14-17 $\mu \mathrm{m}$.

Oedogonium sphaerandrium With \& Lund.

Filamentos cortos, de 10-15 células. Células bicapitadas, con un grosor en los polos de 6-7,5 $\mu \mathrm{m}$ y en el centro de 4-5 $\mu \mathrm{m}$; longitud de 15-20 $\mu \mathrm{m}$. 

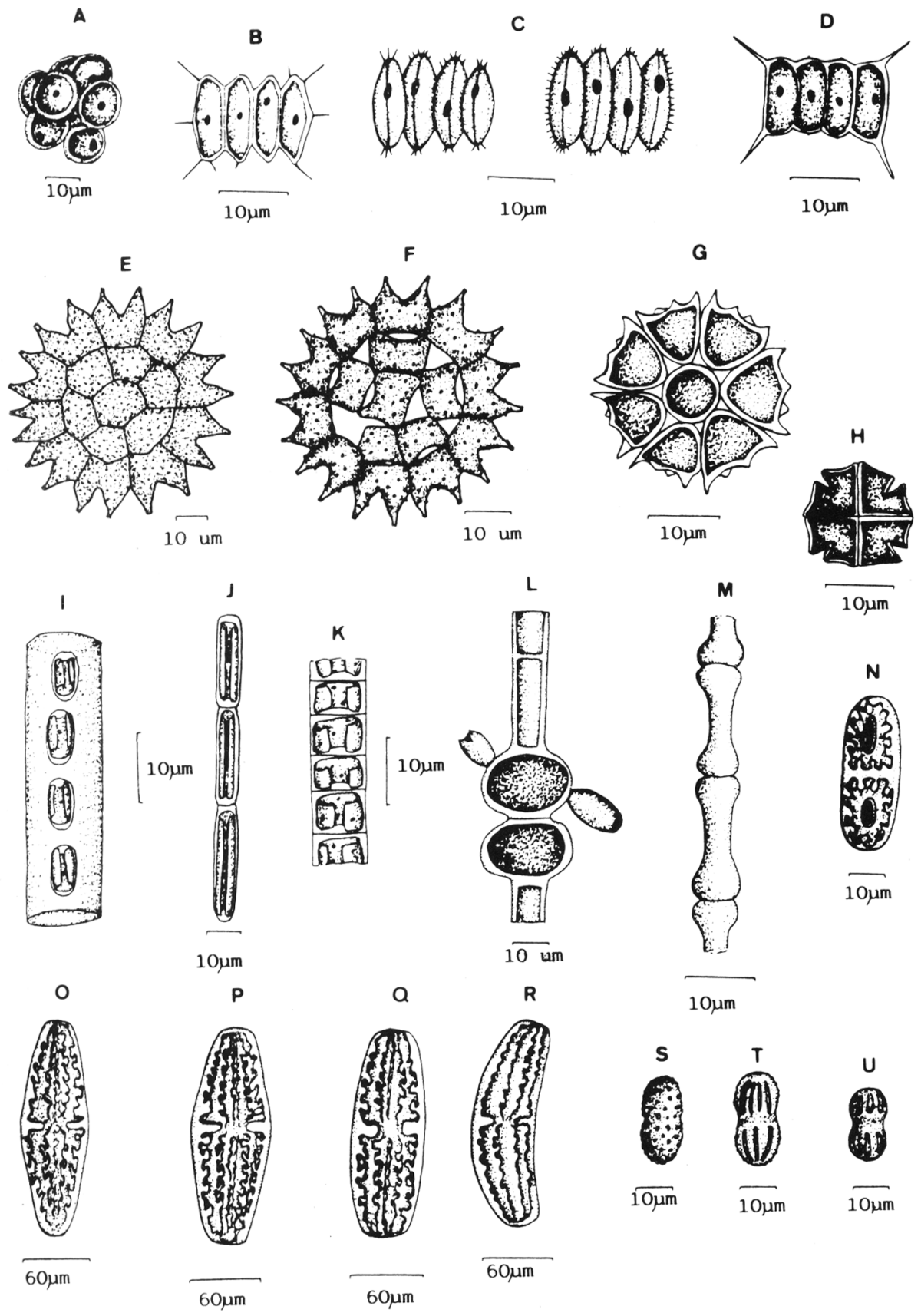

Figura 6.- A. Coelastrum microporum; B, Scenedesmus corallinus; C, Scenedesmus pseudohystrix; D, Scenedesmus quadrispina; E, Pediastrum boryanum; F, Pediastrum boryanum var. cornutum; G, Pediastrum braunii; H, Pediastrum tetras; I, Geminella ordinata; J, Ulothrix subconstricta; K, Ulothrix tenerrima; L, Oedogonium rothii; M, Oedogonium sphaerandrium ; N, Cylindrocystis brebissonii; $0-Q$, Netrium digitus; R, Netrium oblongum var. curvatum; $S-T$, Actinotaenium adelochondrum var. kriegeri; U, Actinotaenium globulosum. 


\section{CLASE ZYGOPHYCEAE}

Familia Mesotaeniaceae

Cylindrocystis brebissonii Menegh.

Fig. 6, N

Células de 2 a 3 veces más largas que anchas. Dimensiones: 43-60 x $17-20 \mu \mathrm{m}$.

Netrium digitus (Ehr.) Itz. et Rothe

Fig. 6, $0-Q$

Células con los extremos truncados y el máximo grosor en el centro, aunque numerosos individuos lo presentan desplazado hacia la zona subapical, observándose también algunas células con los márgenes paralelos en el centro. Dimensiones: 150-210 x (38)48-52 $\mu \mathrm{m}$.

González Guerrero (1950) encuentra ejemplares asimétricos, como los aqui descritos, atribuyendo tal polimorfismo a jóvenes estadíos de crecimiento.

Netrium oblongum (Bory) Lütk. var. curvatum (Fritsch) Koss. $\quad$ Fig. 6, R Células curvadas, con los extremos levemente atenuados y ápices redondeados. Pared muy finamente punteada. Dimensiones: 139-146 x 26-31 $\mu \mathrm{m}$.

\section{Familia Desmidiaceae}

Actinotaenium adelochondrum (Elfv.) Teiling var. kriegeri (Messik.) Ruz.

Fig. 6, S-T

Hemisomas presentando el máximo grosor en el centro, istmo muy poco marcado. La pared presenta gruesos poros muy espaciados, que le dan al margen un aspecto ondulado. Células de 1,7 a 2,2 veces más largas que anchas. Dimensiones: 25-29 x 12-15 $\mu \mathrm{m}$.

Esta variedad, establecida por Messikommer (1942) para los Alpes, se diferencia de la tipo en su menor tamaño y en la forma de los hemisomas, que son redondeados, frente a los claramente truncados de la variedad tipo.

Actinotaenium globosum (Bulnh.) Förster

Pared celular lisa. Dimensiones: 15-20 x 9-11 $\mu \mathrm{m}$.

Closterium lunula (Müll.) Nitzsch ex Ralfs

Fig. 6, U

Dimensiones: 504-600 $\mathrm{m} \times$ 95-100 $\mu \mathrm{m}$.

Closterium strigosum Bréb.

Dimensiones: 178-192(-250) x 14-18(-22) $\mu \mathrm{m}$.

Fig. 7, A

Fig. 7, B

Cosmarium difficile Lütk.

Fig. $7, \mathrm{C}$ Ornamentación muy poco aparente, senos abiertos. Longitud: 31-34 $\mu \mathrm{m}$; anchura: (17-)19-20 $\mathrm{m}$; grosor: $14-15 \mu \mathrm{m}$; istmo: 3,5-5 $\mu \mathrm{m}$.

Cosmarium elegantissimum Lundell

Fig. 7, D

Ornamentación constituida por verrugas longitudinales levemente hundidas en su zona central. Longitud: 48-55 $\mu \mathrm{m}$; anchura y grosor: 21,5-26 $\mu \mathrm{m}$; istmo: 19-20 $\mu \mathrm{m}$.

Las dimensiones observadas coinciden con las dadas por Prescott \& al. (1981) e Hirano (1956) para la forma minor West.

Cosmarium formosulum Hoff.

Fig. 7, E

Longitud: 43-45 $\mu \mathrm{m}$; anchura: 36-38 $\mu \mathrm{m}$; grosor: 26-29 $\mu \mathrm{m}$; istmo: 9,5-11 $\mu \mathrm{m}$. 

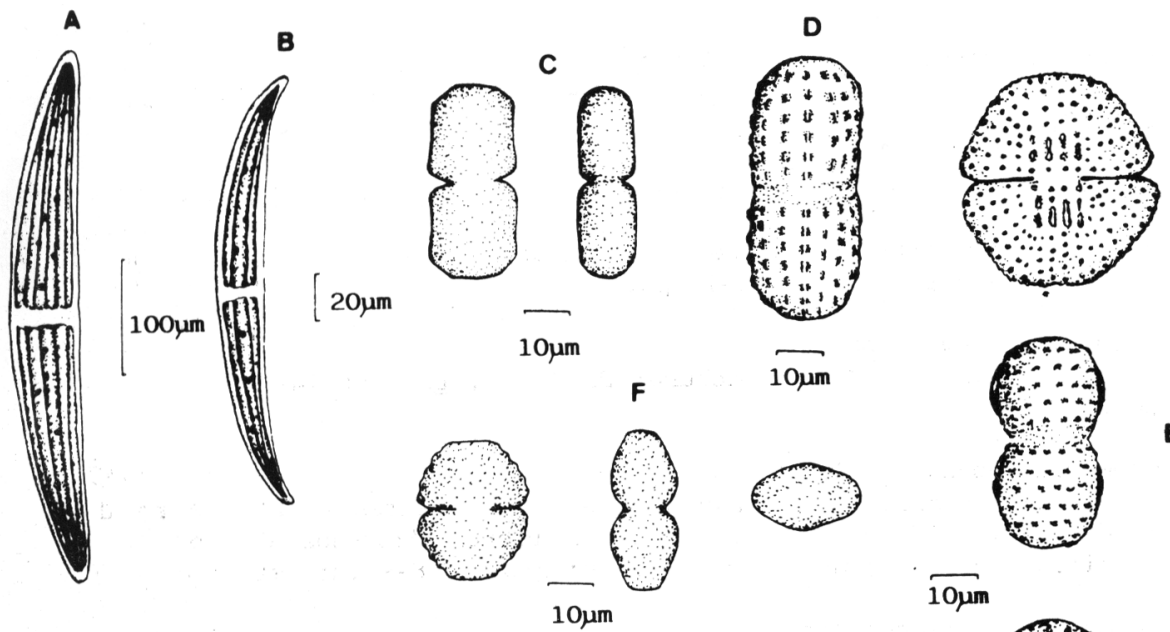

G
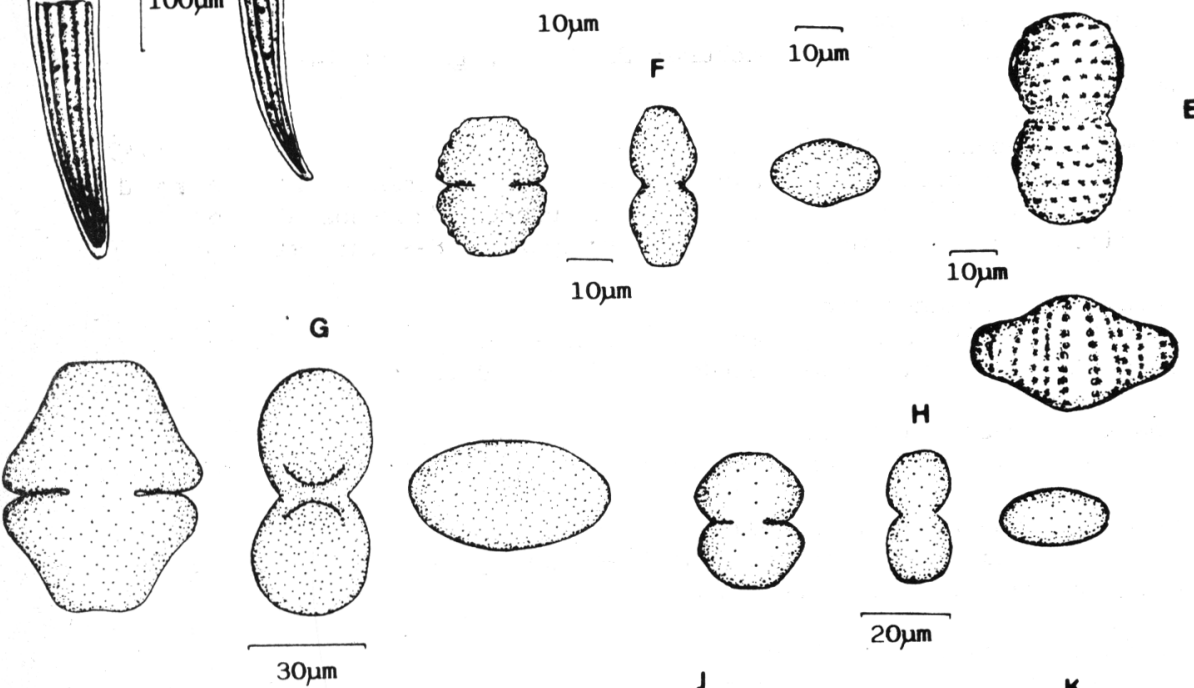

a.s.

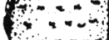

E

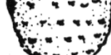

$\widetilde{10 \mu \mathrm{m}}$
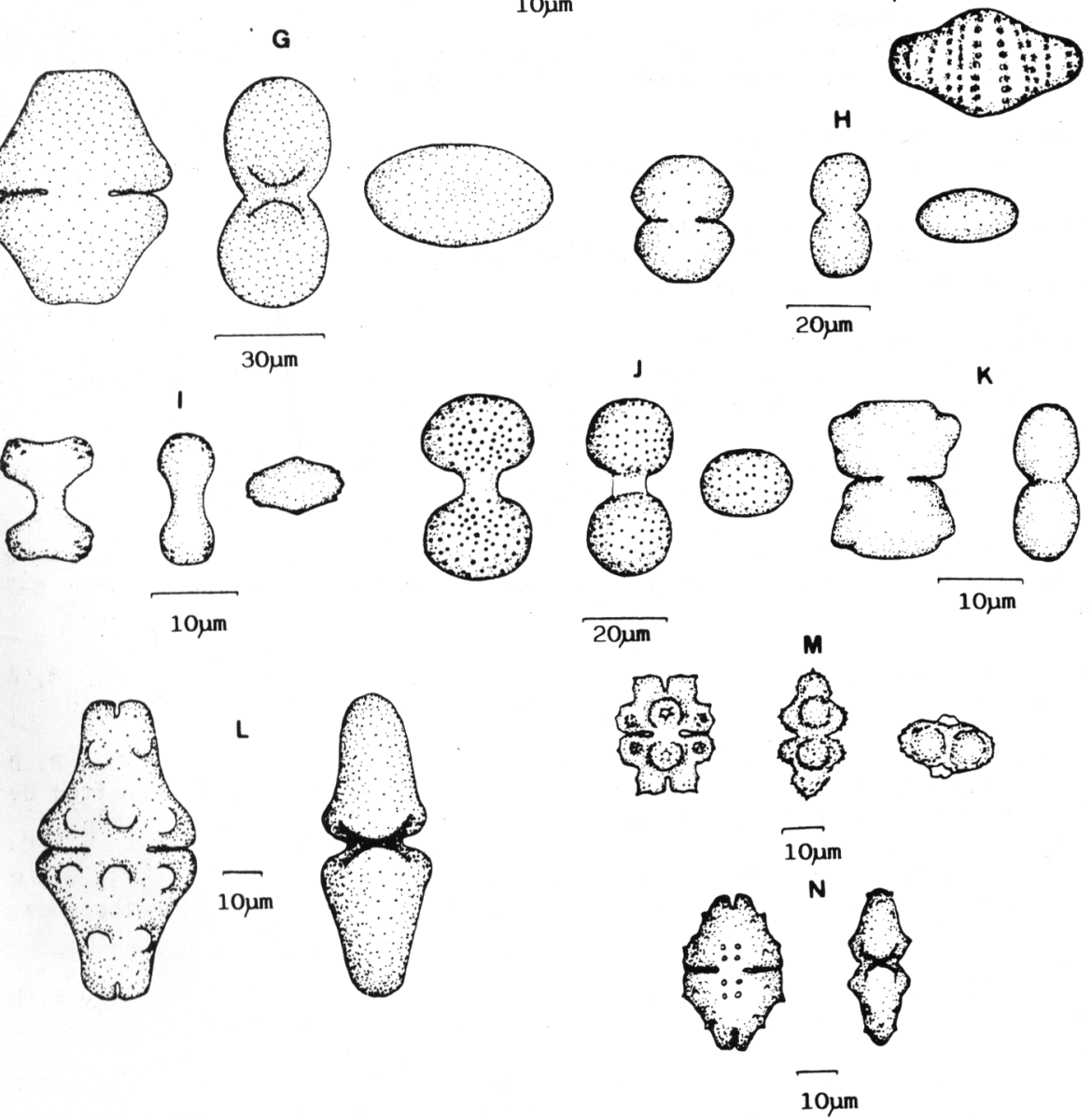

Figura 7.- A, Closterium lunula; B, Closterium strigosum; C, Cosmarium difficile; D, Cosmarium elegantissimum; E, Cosmarium formosulum; F, Cosmarium garrolense; G, Cosmariun hammeri var. homalodermum; H, Cosmarium laeve; I, Cosmarium novae-semliae; J, Cosmariun portianum; K, Cosmarium regnellii; L, Euastrum ansatum; N, Euastrum denticulatum; N, Euastrum elegans. 
Cosmarium garrolense Roy et Bissett Longitud: 26-30 $\mu \mathrm{m}$; anchura: 24-28 $\mu \mathrm{m}$; grosor: 15-17 $\mu \mathrm{m}$; istmo: $8,5-10,5 \mu \mathrm{m}$.

Cosmarium hammeri Reinsch. var homalodermum (Nordst.) W. West et G.S. West

Pared claramente punteada. Longitud: 62-67 $\mu \mathrm{m}$; anchura: 48-53 $\mu \mathrm{m}$; grosor: 31-33 $\mu \mathrm{m}$; istmo: 12-15 $\mu \mathrm{m}$.

Cosmarium laeve Rabh.

Fig. 7, H

Longitud: 29-34 $\mu \mathrm{m}$; anchura: 22-26 $\mu \mathrm{m}$; grosor: 14-17 $\mu \mathrm{m}$; istmo: 6-8 $\mu \mathrm{m}$.

Cosmarium novae-semliae Wille

Fig. 7, I

Ornamentación constituida por pequeños dientes (6-7) que se disponen fundamentalmente en los márgenes superiores externos de los hemisomas. Longitud: 14-15 $\mu \mathrm{m}$; anchura: 11-13 $\mu \mathrm{m}$; grosor: 8-9 $\mu \mathrm{m}$; istmo: 4,5-6,5 $\mu \mathrm{m}$.

Cosmarium portianum Archer

Fig. 7, J Istmo elongado y pared gruesamente punteada. Longitud: 36-41 $\mu \mathrm{m}$; anchura: 25-27 $\mu \mathrm{m}$; grosor: 19-21 $\mu \mathrm{m}$; istmo: 9-10 $\mu \mathrm{m}$.

Cosmarium regnellii wille Longitud: 15-17 $\mu \mathrm{m}$; anchura: 14-15 $\mu \mathrm{m}$; grosor: 8-9 $\mu \mathrm{m}$; istmo: 4-5 $\mu \mathrm{m}$.

Euastrum ansatum Ehr. ex Ralfs

Fig. 7, L Longitud: 72-89 $\mu \mathrm{m}$; anchura: 38-45 $\mu \mathrm{m}$; grosor: 25-29 $\mu \mathrm{m}$; istmo: 12-15 $\mu \mathrm{m}$; anchura del ápice: 15-17 $\mu \mathrm{m}$.

Euastrum denticulatum (Kirch.) Gay

Fig. 7, M Longitud: 25-31 $\mu \mathrm{m}$; anchura: 21-22 $\mu \mathrm{m}$; grosor: 15-17 $\mu \mathrm{m}$; istmo: 5-6 $\mu \mathrm{m}$; anchura de los lóbulos apicales: 9-10 $\mu \mathrm{m}$. La incisión apical, así como la forma de los ángulos constituidos por los lóbulos laterales y apicales, coinciden con los de ls población encontrada por Caballero (1950) en la Sierra de Gredos.

Euastrum elegans (Bréb.) Ralfs

Fig. $7, \mathrm{~N}$ Longitud: 33-36 $\mu \mathrm{m}$; anchura: 21-24 $\mu \mathrm{m}$; grosor: 11-13 $\mu \mathrm{m}$; istmo: 6-7 $\mu \mathrm{m}$.

Euastrum oblongum (Grev.) Ralfs

Fig. 8, A

Longitud: 119-156 $\mu \mathrm{m}$; anchura: 71-89 $\mu \mathrm{m}$; istmo: 17-24 $\mu \mathrm{m}$.

Euastrum verrucosum Ehr. ex Ralfs

Fig. 8, B

Longitud: 90-94 $\mu \mathrm{m}$; anchura: 78-84 $\mu \mathrm{m}$; grosor: 53-57 $\mu \mathrm{m}$; longitud de los lóbulos subapicales: 7-9 $\mu \mathrm{m}$.

Hyalotheca dissiliens (Smith) Bréb. ex Ralfs

Fig. 8, C Las células presentan de 4-6 filas horizontales de granulaciones. Longitud: 22-24 $\mu \mathrm{m}$; anchura: 19-23 $\mu \mathrm{m}$.

Pleurotaenium trabecula Ehr. ex Näg. Fig. 8, D Longitud: 400-475 $\mu \mathrm{m}$; anchura: 41-43 $\mu \mathrm{m}$; istmo: $35-37 \mu \mathrm{m}$.

Spondylosium planum (Wolle) W. West et G.S. West Fig. 8, E Longitud: 13-17 $\mu \mathrm{m}$; anchura: 11-13 $\mu \mathrm{m}$; grosor: 7-9 $\mu \mathrm{m}$; istmo: 5-6 $\mu \mathrm{m}$.

Staurastrum capitulum Bréb. Longitud: 36-40 $\mu \mathrm{m}$; anchura: 21-26,5 $\mu \mathrm{m}$; istmo: 12-17 $\mu \mathrm{m}$. 

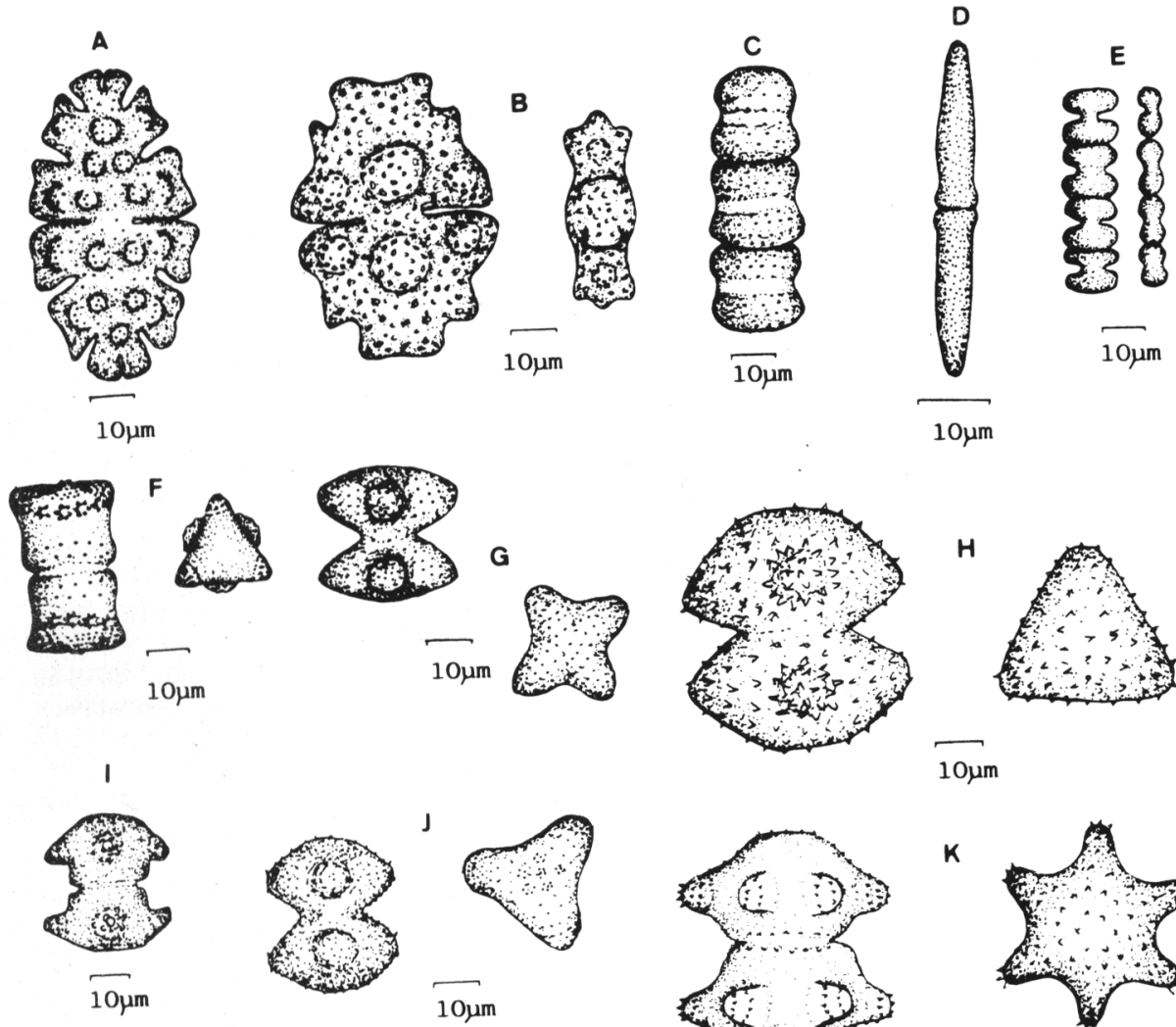

Oum
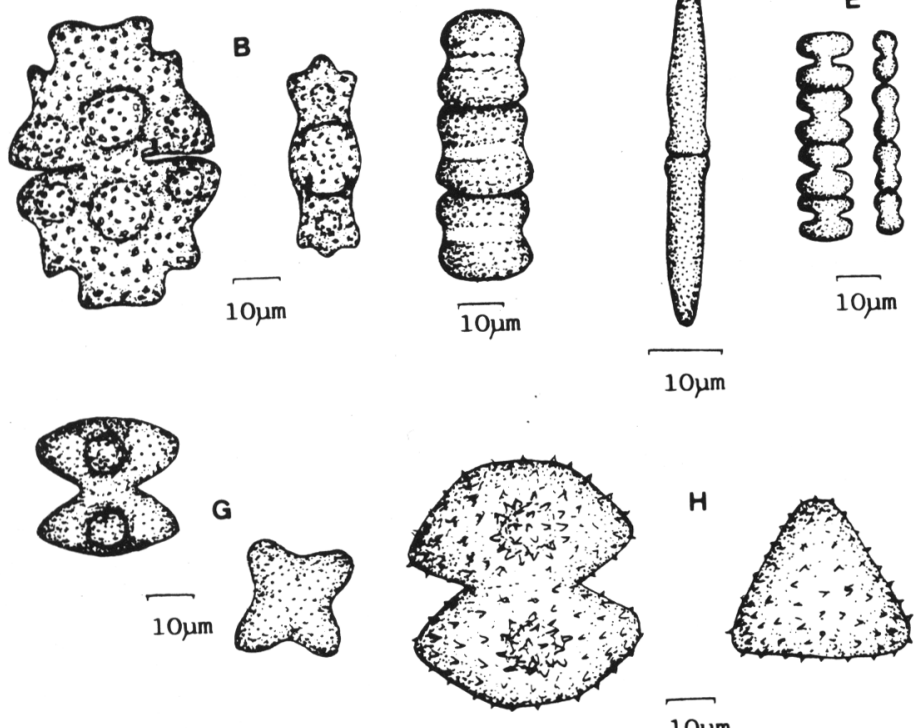

$\widetilde{10 \mu \mathrm{m}}$
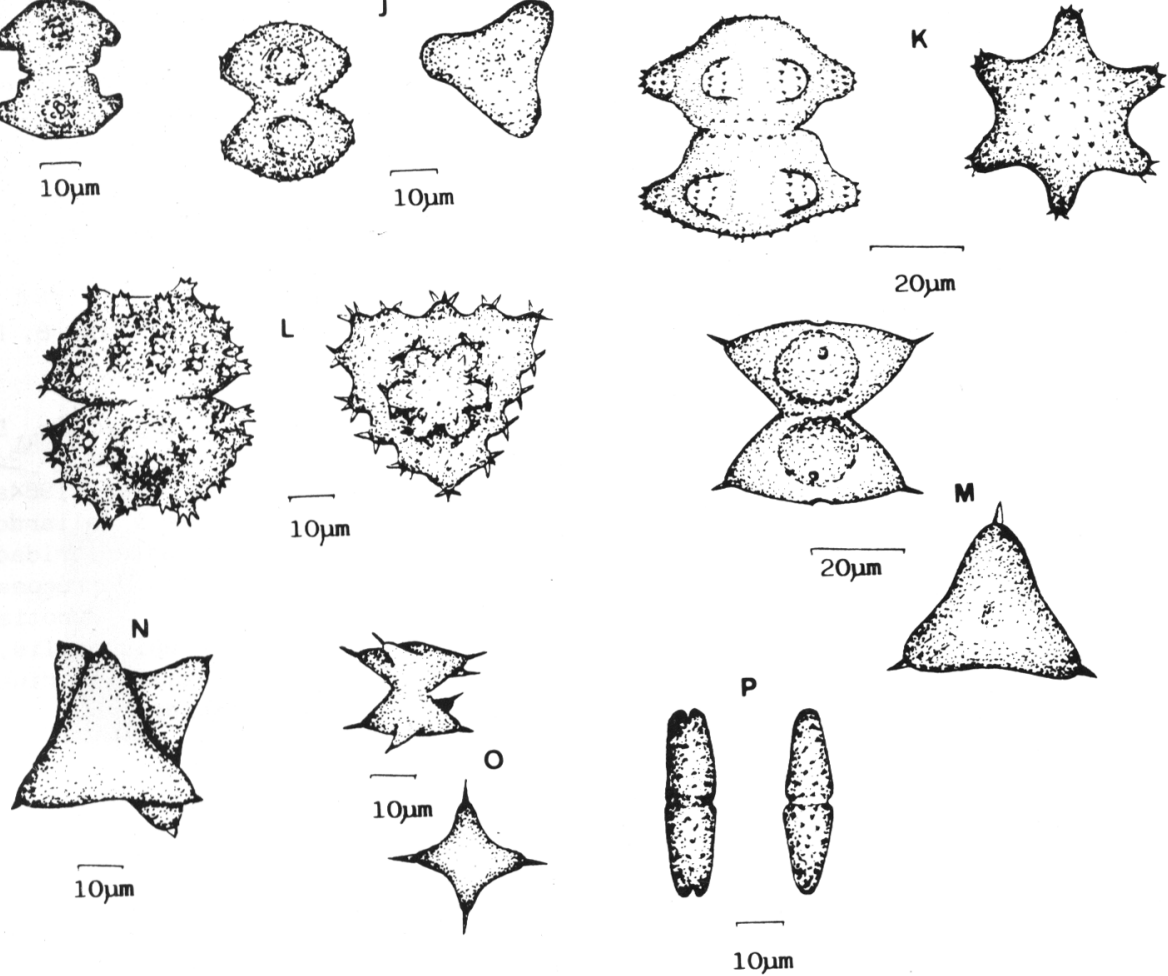

Figura 8.- A, Euastrum oblongum; B, Euastrum verrucosum; C, Hyalotheca dissiliens; D, Pleurotaenium trabecula; E, Spondylosium planum; F, Staurastrum capitulum; G, Staurastrum dilatatum; H, Staurastrum hirsutum; I, Staurastrum margaritaceum; J, Staurastrum punctulatum; K, Staurastrum sexcostatum; L, Staurastrum spongiosum var. perbifidum; M-N, Staurodesmus dejectus (N, forma "janus"); 0 , Staurodesmus spencerianus; P, Tetmemorus laevis. 
Staurastrum dilatatum (Ehr.) Ralfs

Longitud: 26-29 $\mu \mathrm{m}$; anchura: 29-31 $\mu \mathrm{m}$; istmo: 10-12 $\mu \mathrm{m}$.

Fig. 8, G

Staurastrum hirsutum (Ehr.) Ralfs

Longitud: 43-45 $\mu \mathrm{m}$; anchura: $40-46 \mu \mathrm{m}$; istmo: 15-18 $\mu \mathrm{m}$.

Fig. 8, H

Staurastrum margaritaceum (Ehr.) Ralfs

Longitud: 28-30 $\mu \mathrm{m}$; anchura: 25-27 $\mu \mathrm{m}$; istmo: 11-12 $\mu \mathrm{m}$.

Fig. 8, I

Staurastrum punctulatum (Bréb.) Ralfs

Longitud: 29-34 $\mu \mathrm{m}$; anchura: 26-29 $\mu \mathrm{m}$; istmo: 9-10 $\mu \mathrm{m}$.

Fig. 8, J

Fig. 8, K

Staurastrum sexcostatum (Bréb.) Ralfs

Longitud: 43-47,5 $\mu \mathrm{m}$; anchura: 43-47,5 $\mu \mathrm{m}$; istmo: 15-19 $\mu \mathrm{m}$.

Las poblaciones estudiadas se podrían incluir en la var. productum

West, por la longitud de los procesos y distribución de espinas, pero las dimensiones celulares son más próximas a las de la variedad tipo.

Staurastrum spongiosum Bréb. ex Ralfs var. perbifidum West Fig. 8, L Longitud: 55-60 $\mu \mathrm{m}$; anchura: 43-50 $\mu \mathrm{m}$; istmo: 15-19 $\mu \mathrm{m}$.

Tanto la longitud como la anchura del istmo, son mayores que en las descripciones consultadas, a excepción de la de Margalef (1956), que ofrece las siguientes: longitud: 86-92 $\mu \mathrm{m}$; anchura: 50-52 $\mu \mathrm{m}$; istmo: $25 \mu \mathrm{m}$.

Staurodesmus dejectus (Bréb.) Teiling

Fig. 8, M-N Hemisomas normalmente trilobulados. Dimensiones celulares, longitud: 36-41 $\mu \mathrm{m}$; anchura: 36-43 $\mu \mathrm{m}$; istmo: 9-10 $\mu \mathrm{m}$.

En algunas ocasiones se han observado formas "Janus", con hemisomas tri- y tetralobulados, alcanzando el hemisoma tetralobulado hasta $48 \mu \mathrm{m}$ de anchura.

Staurodesmus spencerianus (Mask.) Teiling

Fig. 8, 0 Longitud: 24-26 $\mu \mathrm{m}$; anchura: 24-25 $\mu \mathrm{m}$; istmo: 9-10 $\mu \mathrm{m}$; longitud de las espjnas: $4-6(-10) \mu \mathrm{m}$.

Tetmemorus laevis (Kütz.) Ralfs

Longitud: 62-79 $\mu \mathrm{m}$; anchura: 20-23 $\mu \mathrm{m}$.

Fig. 8, P

De los 149 taxones estudiados, 21 son Cyanophyceae, 2 Dinophyceae, 8 Euglenophyceae, 5 Chrysophyceae, 51 Diatomophyceae, 28 Chlorophyceae y 34 Zygophyceae. Según la recopilación bibliográfica de Alvarez Cobelas (1984a y b). Alvarez Cobelas \& Estévez García (1982) y Alvarez Cobelas \& Gallardo (1986), 11 de los taxones encontrados no se han citado con anterioridad para nuestra ficoflora: Trachelomonas acanthostoma, Trachelomonas verrucosa var. macrotuberculata, Fragilaria pinnata var. trigona, Eunotia pseudopectinalis, Neidium affine var. longiceps, Pinnularia episcopalis, Scotiella tuberculata, Eutetramorus fotii, Scenedesmus corallinus, Netrium oblongum var. curvatum y Actinotaenium adelochondrum var. kriegeri.

\section{DISCUSION}

En la tabla 1 se muestra la distribución de las especies en el conjunto de las lagunas estudiadas. Se ha de señalar, como ya se indicó en la introducción, que el estudio de las 10 primeras lagunas se basa en las observaciones llevadas a cabo en un periodo libre de hielo, mientras que las 6 últimas sólo se muestrearon esporádicamente, por lo que determinadas 
diferencias florísticas han de atribuirse, más que a una distinta composición de su ficoflora, al incompleto conocimiento que aún se posee de la misma.

De especial interés biogeográfico son algunos de los hallazgos encontrados en dos de las lagunas estudiadas: Cuarta y Hondera. En la primera son varias especies de diatomeas y desmidiaceas las que centran nuestro interés; entre las diatomeas se pueden destacar Eunotia pseudopectinalis y E. sudetica. La primera por los escasos testimonios que de ella existen en la bibliografia consultada, ya que sólo se conoce su presencia en la península de Escandinavia (Hustedt, 1942, 1959), a excepción de la var. vasconiensis encontrada en el sur de Francia (Allorge \& Manguin, 1941). E. sudetica, sin embargo, parece ser un taxon con abundante representación en las altas montañas, aunque no exclusivo de ellas. En los sistemas montañosos ibéricos ha sido localizada en los Pirineos (Carter, 1970, Margalef, 1948, 1952), en Guadarrama (Marga1ef, 1949) y en Segundera (Margalef, 1956); a nivel europeo se pueden apuntar sus citas en los Alpes (Messikommer, 1957; Wuthrich, 1975). Entre las desmidiaceas se puede destacar a Cosmarium elegantissimum, que también fue citada en los Pirineos (Allorge \& Manguin, 1941), su adaptación a los hábitats alpinos queda reflejada en las citas de los Alpes (Messikommer, 1942, 1957) y de los Apeninos (De11'Uomo, 1978).
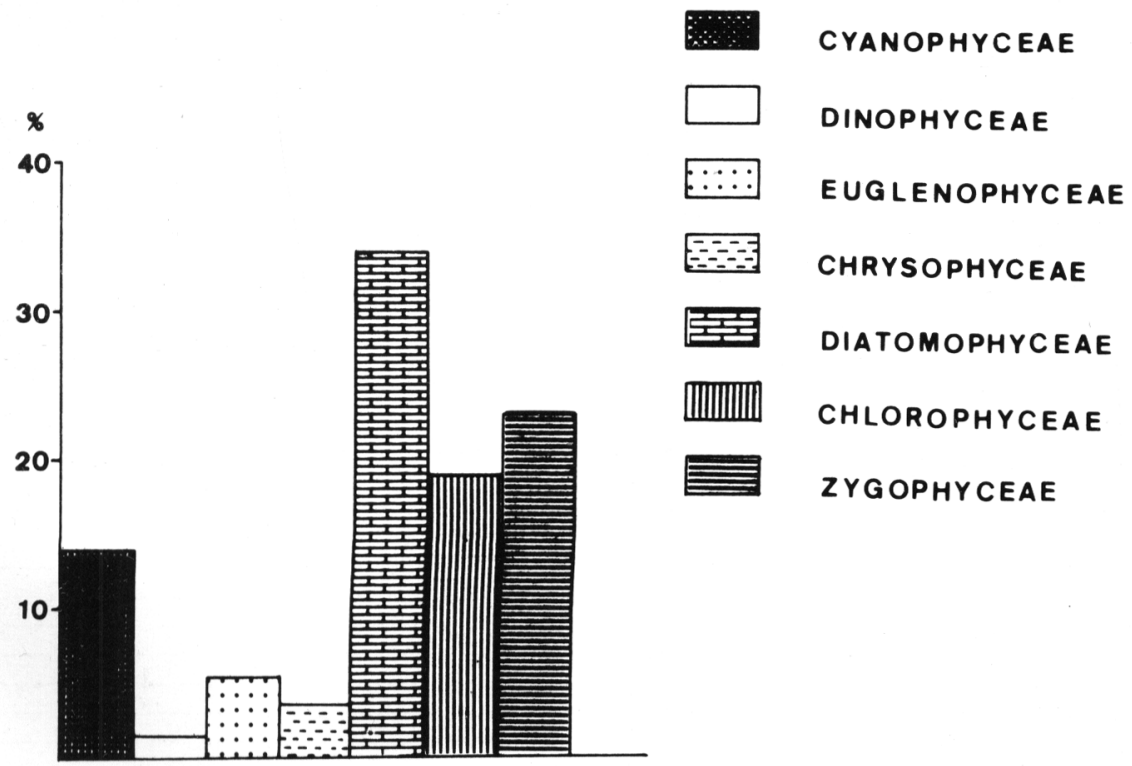

Figura 9.- Representación porcentual de las distintas clases que componen el fitoplancton de las lagunas de Sierra Nevada.

En la laguna Hondera destacan 3 taxones: Netrium oblongum var. curvatum, Actinotaenium adelochondrum var. kriegeri y Staurastrum capitulum, de los cuales sólo el último se conoce de otros macizos montañosos peninsulares: Pirineos (Allorge \& Manguin, 1941; Margalef, 1948); Gerês (González Guerrero, 1950) y Gredos (Caballero, 1944), estando recogida su presencia en 
Aphanothece icroscopica Chroococcus inor Chroococcus tenax Chroococcus turgidus Cyanarcus sp. Eucapsis alpina Gomphosphaeria lacustris Merismopedia punctata Microcystis holsatica Microcystis marginata Synechococcus maior Synechococcus sp. Stigonema ocellatum Anabaena cylindrica Anabaena lapponica Nostoc kihlmani i Oscillatoria agardhii Oscillatoria amoena Oscillatoria curviceps Oscillatoria planctonica Oscillatoria splendida Peridiniun unbonatua Peridinium willei Euglena spirogyra Phacus acuminatus Phacus lemmermanni i Irachelomonas abrupta var. minor

Irachelomonas acanthostoma Trachelomas hispida Irachelomonas lacustris Trachelomonas verrucosa var. acrotuberculata Chromulina ikroplankton Chromulina minima Chromulina nevadensis Chromulina ovaloides Chromulina rosanoffi Cyclotella menghiniana Diatona hienale

var. mesodon

Fragilaria pinnata

var. pinnata

var. trigona

Fragilaria virescens

Hannaea arcus

Meridion circulare

Synedra rumpens

Tabellaria flocculosa

Eunotia biggiba

Eunotia lunaris

Eunotia pectinalis

var. ainor

Eunotia pseudopectinalis Eunotia sudetica

Achnanthes minutissima Achnanthes lanceolata Achnanthes linearis

var. pusilla

Cocconeis placentula

var. lineata

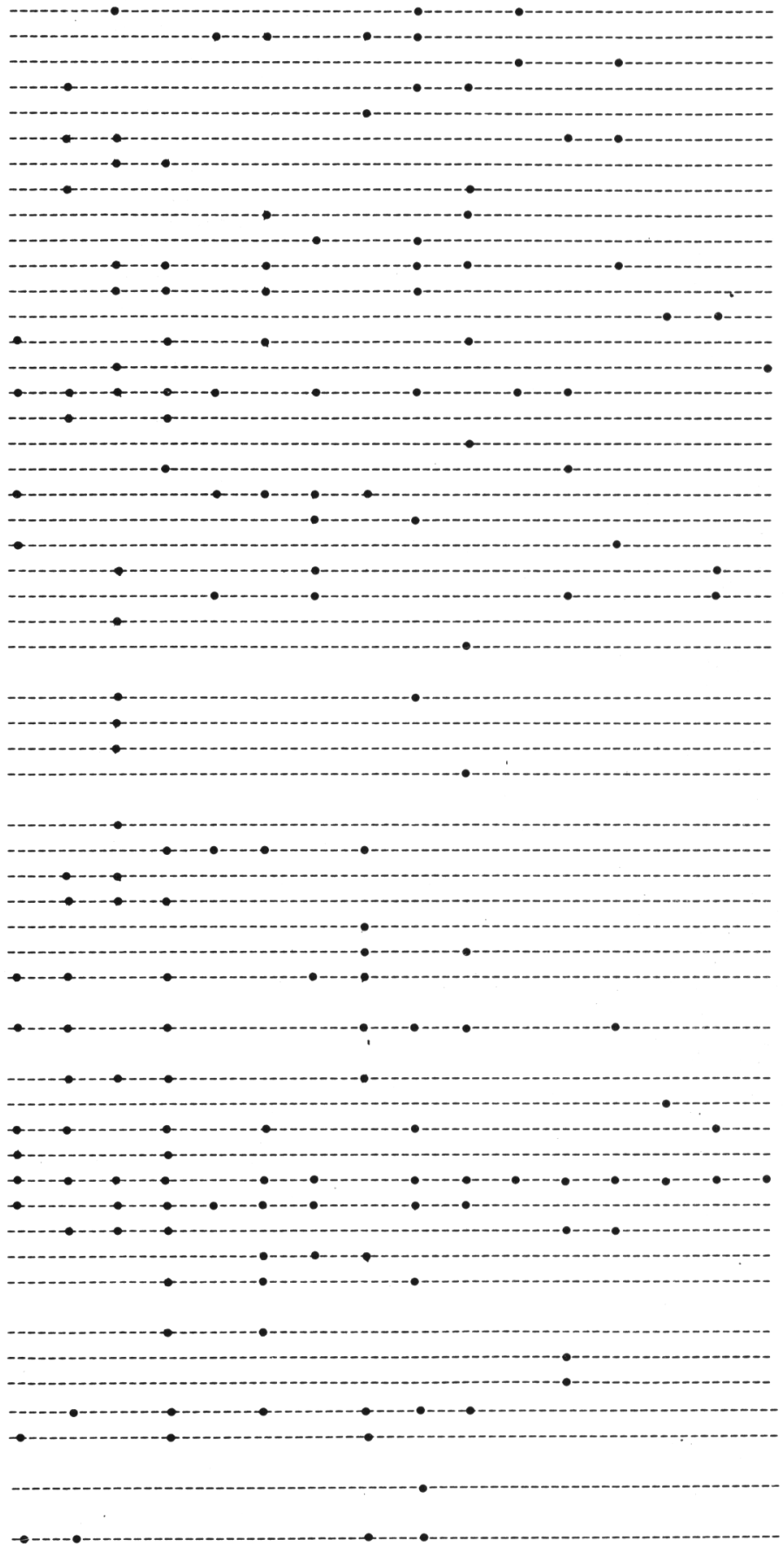


Algas de Sierra Nevada

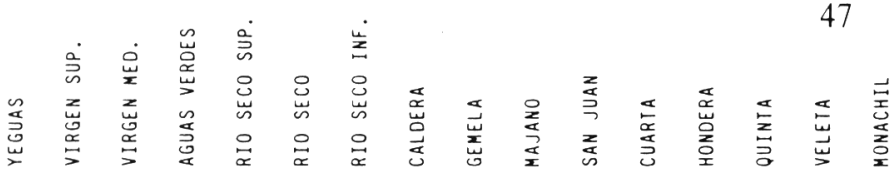

Anomoeoneis serians

var. brachysir

Caloneis ventricosa

Frustulia rhomboides

Navicula cocconeiformis

Navicula cryptocephala

Navicula laevissima

Navicula pseudoscutiformis

Navicula radiosa

Navicula rhynchocephala

Neidiu affine

var. longiceps

Neidiu bissulcatum

Neidium iridis

var. ampliatum

Pinnularia borealis

Pinnularia episcopalis

Pinnularia hemiptera

Pinnularia microstauron

Pinnularia viridis

Stauroneis anceps

Stauroneis phoenicenteron

Stauroneis soithii

Gomphonena affine

Gomphonema longiceps

Amphora ovalis

Cymbella aspera

Cymbella gracilis

Cymbel!a minuta

Cymbella naviculiformis

Cymbella parva

Cymbella subaequalis

Nitzschia hantzschiana

Surirella angustata

Surirella linearis

Surirella tenera

Chlamydomonas microsphaerella

Polytona angustum

Pandorina morum

Tetraspora lemnermannit

Chlanydocapsa planctonic.

Chlorangiella pygmaea

Korshikoviella gracilipes

Tetraedron caudatum

Ankistrodesnus falcatus

Oocystis lacustris

Oocyst is marssoni

0ocystis solitaria

Scotiella tuberculata

Eutetramorus fotti

Actinastrum hantzschii

Coelastrun microporun

Scenedesaus corallinus

Scenedesaus pseudohystrix

Scenedesaus quadrispina

Pediastrum boryanum

Pediastrum boryanum

var. cornutu

Pediastrum brauni

Pediastrum tetras
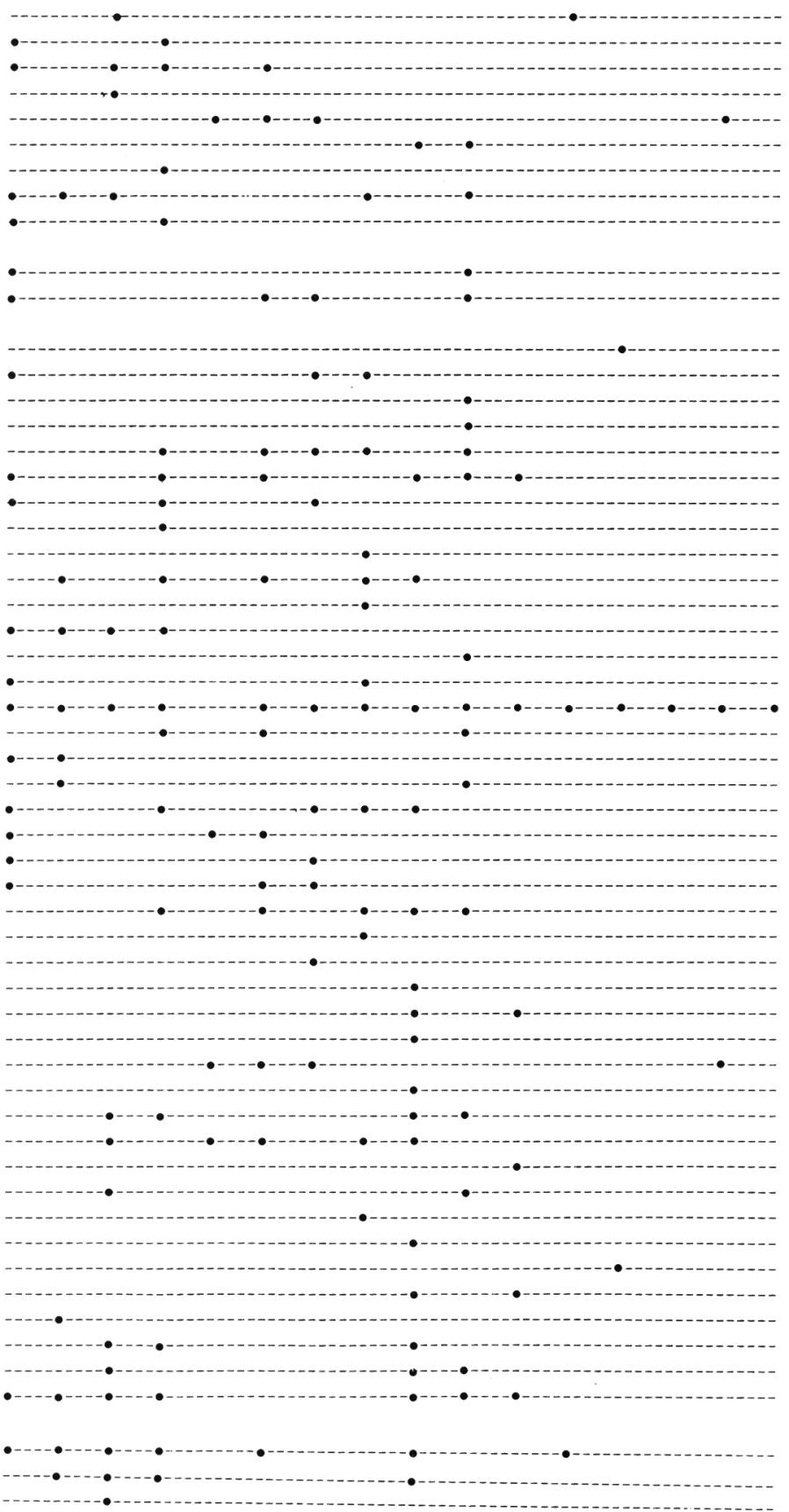

TABLA 1 (Continuación).- Presencia de las especies en las lagunas estudiadas. 
Geminella ordinata Ulothrix subconstricta Ulothrix tenerrima Oedogonium rothii 0edogoniun sphaerandrium Cylindrocystis brebissonii Netrium digitus Netrium oblongu var. curvatum Actinotaenium adelochondrua var. kriegeri

Actinotaenium globosum Closteriua lunula Closterium strigosum Cosmariun difficile Cosmarium elegantissinua Cosmariun formosulua Cosmarium garrolense Cosmarium hammeri var. homalodermum Cosmariun laeve Cosmariun novae-semliae Cosmarium portianum Cosnariun regnelli Euastrum ansatum Euastrum denticulatum Euastrum elegans Euastrum oblongum Euastrum verrucosum Hyalotheca dissiliens Pleurotaenium trabecula Spondilosium planum Staurastrum capitulum Staurastrum dilatatum Staurastrum hirsutum Staurastrum argaritaceum Staurastrum punctulatum Staurastrum sexcostatum Staurastrun spongiosum var. perbifidun Staurodesmus de jectus Staurodesmus spencerianus Tetmemorus laevis

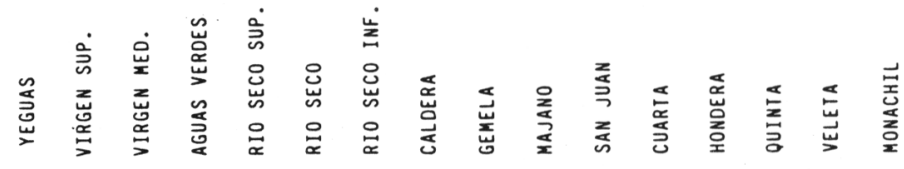
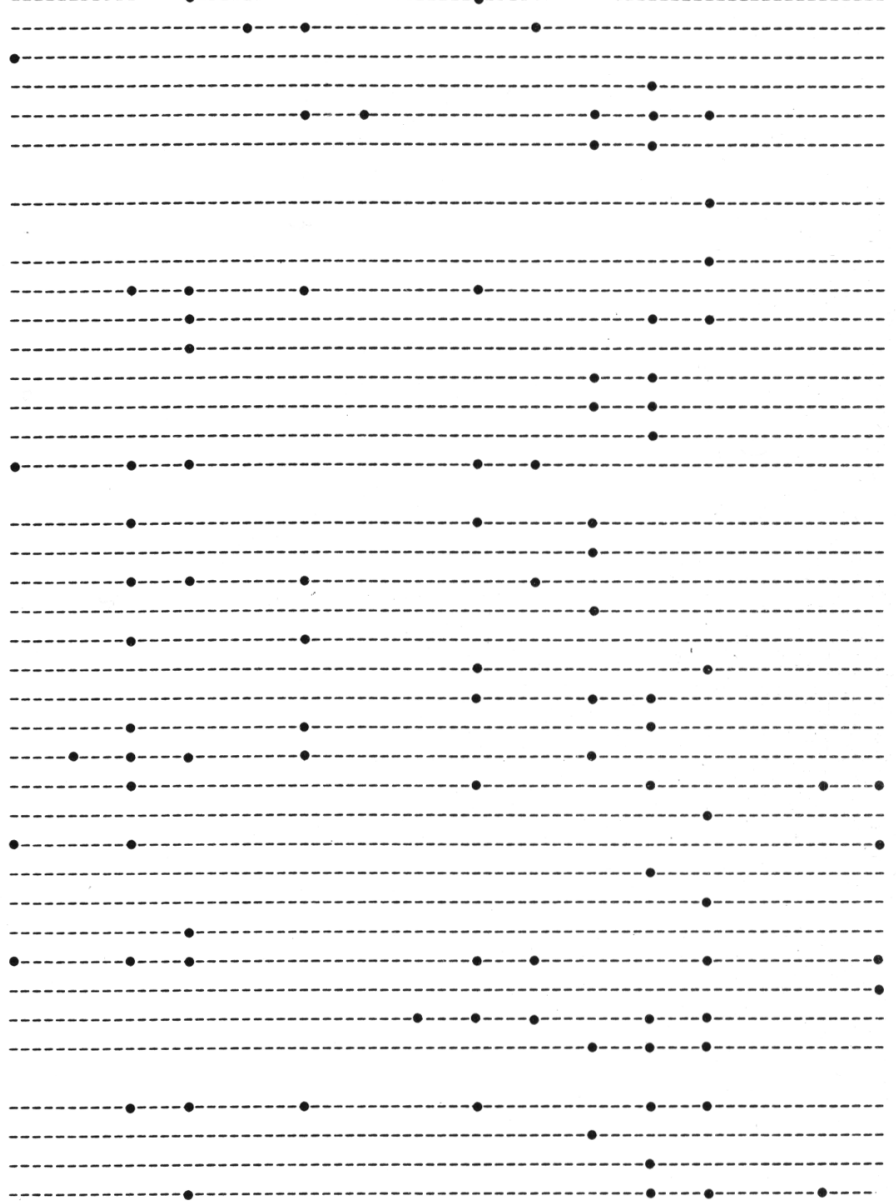

IABLA 1 (Continuación).- Presencia de las especies en las lagunas estudiadas. 
otras montañas europeas, entre las que podemos destacar los Alpes (Deflandre, 1925; Messikommer, 1942, 1957) y las montañas de Córcega (Guinochet, 1936).

Además de las especies comentadas, se pueden destacar como elementos de distribución ártico-alpina a Pediastrum braunii y Eunotia bigibba, localizadas en la laguna Virgen Media la primera y en las de La Caldera, Río Seco y Rio Seco Inferior la segunda.

En la figura 9 se obșerva la representación de cada uno de los grupos éstudiados en el conjunto de las lagunas de alta montaña de Sierra Nevada. De esta forma se puede apreciar que son los diatomeas el grupo mejor representado, ya que constituyen el $34 \%$ del total de las especies estudiadas, siendo las lagunas de Las Yeguas, Virgen Superior, Aguas Verdes, Río Seco Inferior y Río Seco las que destacan por su mayor representción de este grupo de algas. Las especies que se presentan de forma más asidua en el conjunto de las lagunas son: Meridion circulare y Cymbella minuta; la primera caracteristica de aguas frías y bien oxigenadas, por lo que alcanza su mejor representación en las aguas corrientes de alta montaña (Sánchez Casti11o, 1984), mientras que la segunda especie presenta una gran amplitud ecológica, siendo muy frecuente en cualquier tipo de aguas.

A continuación los grupos con mejor representación son zigoficeas y cloroficeas, con el 23 y el $19 \%$ del porcentaje total. Ambas clases parecen estar mejor representadas en aquellas lagunas poco profundas y con prados inundados en su perisferia, hábitat muy propicio para el desarrollo de ambos grupos. Entre las 1 agunas muestreadas periódicamente son Virgen Media, Aguas Verdes, Gemela y Majano las que ofrecen una mayor representación, siendo Pediastrum boryanum, Cosmarium garrolense y Staurastrum hirsutum las especies más constantes en la mayoría de las lagunas. Entre las lagunas no muestreadas periódicamente destacan las de San Juan, Cuarta y Hondera por su gran proporción de zigoficeas.

Las cianofíceas representan el $14 \%$ del total, siendo Nostoc kihlmani la especie que muestra una mayor presencia en las lagunas estudiadas. E1 resto de los grupos estan desigual y pobremente representados en el conjunto de las lagunas.

\section{AGRADECIMIENTOS}

Al Dr. J. Varo, director de mi tesis doctoral, de la que se ha extraído este catálogo; al Dr. P. Coesel por su amable revisión de determinados taxones de desmidiáceas y el Dr. P. Simonsen por habernos proporcionado las microfotografías del material tipo de Eunotia pseudopectinalis.

\section{BIBLIOGRAFIA}

ALLORGE, P. \& MANGUIN, E. -1941- Algues d'eau douce des Pyrenées basques, Bull. Soc. Bot. France, 88:159-191.

ALVAREZ COBELAS, M. -1984a- Catálogo de las algas continentales españolas, II. Craspedophyceae, Cryptophyceae, Chrysophyceae, Dinophyceae, Euglenophyceae, Haptophyceae, Phaeophyceae, Rodophyceae y Xanthophyceae. Acta Bot. Malacitana, 9:27-40. 
ALVAREZ COBELAS, M. -1984b- Catálogo de las algas continentales españolas, III, Zygophyceae Widder 1960. Coll. Bot., 15:17-37.

ALVAREZ COBELAS, M. \& ESTEVEZ GARCIA, A. -1982- Catálogo de las algas continentales españolas, I. Diatomophyceae Rabenjorst 1864. Lazaroa, $4: 269-285$.

ALVAREZ COBELAS, M \& GALLARDO, T. -1986- Catálogo de las algas continentales españolas, IV. Chlorophyceae Wille in Warning 1884. Prasinophyceae T. Christensen ex Silva 1980. Acta Bot. Malacitana, $11: 17-38$.

BACHMANN, A. -1913- Planktonproben aus Spanien, gesammelt von Prof. Halbfab. Berich. Deutsch. Bot. Gesellschaft, 13:183-188.

BELLOC, E. -1892- Aparçu général de la vegetation lacustre dans les Pyrénées. Assoc. Française pour le avancement des Sciences. Congres de Pau: 88-92.

BOURRELly, P. -1970- Les algues d'eau douce. Algues bleues et rouges, 512 pp. Ed. Boubée et Cie. Paris.

CABALlero, F. -1944- Algas del macizo de Gredos. Anales Jard. Bot. Madrid, $5(2): 345-364$.

CABAllero, F. -1950- Algas del macizo de Gredos ( 2 a parte). Anales Jard. Bot. Madrid, 10(1):231-260.

CARTER, J.R. -1970- Diatoms from Andorra. Nova Hedwigia, 31:605-632.

DEFLANDRE, G. -1925- Note sur la flore algologique de deux localités alpines. Bull. Soc. Bot. France, 72:373-393.

DELL'UOMO, A. -1978- Alcune Desmidiaceae raccolte nella torbiera del Tonale. Studi Trentini di Scienze Naturali, 55:11-15.

GEITLER, L. -1932- Cyanophyceae In: Dr. L. Rabenhorst's Kryptogamen-Flora. 1196 pp. Leipzig.

GERMAIN, H. -1981- Flore des Diatomées. 444 pp. Ed. Boubée. Paris.

GONZALEZ GUERRERO, P. -1927- Contribución al conocimiento ficológico del Pirineo español. Bol. R. Soc. Esp. Hist. Nat., 27:343-346.

GONZALEZ GUERRERO, P. -1929a- Nuevos datos sobre el plancton hispano-marroquí I. Bol. R. Soc. Esp. Hist. Nat., 29:251-254.

GONZALEZ GUERRERO, P. -1929b- Nuevos datos sobre el plancton hispano-marroquí II. Bol. R. Soc. Esp. Hist. Nat., 29:361-364.

GONZALEZ GUERRERO, P. -1942- Algas del Norte y Centro de España. Anales Jard. Bot. Madrid, 3:269-278.

GONZALEZ GUERRERO, P. -1950- Algas y cianofíceas de la Sierra de Geres. Agronomía lusitánica, 12:15-79.

GONZALEZ GUERRERO, P. -1965- Algas de la silice (Guadarrama). Anales Jard. Bot. Madrid, 23:93-110.

GONZALEZ GUERRERO, P. -1975- Los ficotopos de la alta Alpujarra (Granada). Trab. Dept. Bot. Univ. Granada, 3(1):61-85.

GONZALEZ GUERRERO, P. -1976- Los ficotopos de la alta Alpujarra (Continuación). Trab. Dept. Bot. Univ. Granada, 3(2):163-201.

GUINOCHET, M. -1936- Algues d'eau douce récoltées au cours de la session de la Societé Botanique de France en Corse (août 1930). Bull. Soc. Bot. France, 83:508-524.

HIRANO, M. -1956- Flora Desmidiacearum Japonicarum. II. Contr. Biol. Labor. Kyoto Univ., 2:57-106.

HIRANO, M. -1972- Diatoms from the Mountain Range in the Japan Alps. Contr. Biol. Labor. Kyoto Univ., 24(1):9-30.

HIRANO, M. -1983- Fresh-water algae from Bhutan. Study Reports of Baika Junior College, 31:171-183.

HUBER-PESTALOZZI, G. -1955- Das Phytoplankton des Süsswassers, T. 4. Euglenophyceen $606 \mathrm{pp}$. Stuttgart.

HUSTEDT, F. -1942- Diatomeen aus der Umgebung von Abisko in Schwedisch-Lappland. Archiv für Hydrobiologie, 39(1):87-174.

HUSTEDT, F. -1959- Die Kieselalgen (2). 845 pp. In: Dr. Rabenhorst's Kryptogamen-Flora. Bremen. 
HUSTEDT, F. -1961/1966- Die kieselalgen (3). 816 pp. In: L. Rabenhorst's kryptogamen-Flora. Bremen.

MARGALEF, R. -1948- Flora, fauna y comunidades bióticas de las aguas dulces del Pirineo de la Cerdeña. Estación de Estudios Pirenaicos, 11:1-197.

MARGALEF, R. -1949- Datos para la hidrobiología de la Sierra de Guadarrama. Publ. Inst. Biol. Apl., 6:5-21.

MARGALEF, R. -1950- Datos para la hidrobiología de la Cordillera Cantábrica, especialmente del macizo de los Picos de Europa. Publ. Inst. Biol. Apl., 7:37-76.

MARGALEF, R. -1952- La vida en las aguas dulces de Andorra. 107 pp. Primer Congreso Internacional del Pirineo. Zaragoza.

MARGALEF, R. -1953- La vida en los lagos de alta montaña de los Pirineos. Pirineos, 14:5-31.

MARGALEF, R. -1956- Algas de agua dulce del NW de España. Publ. Inst. Biol. Apl., 22:43-152.

MARGALEF, R., VILASECA, J.M., CAMPAS, L. \& MIRACLE, M.R. -1975- Introdución al estudio de los lagos pirenaicos. Naturalia Hispanica, 5:1-47.

MARTINEZ, R. -1975- First report on the limnology of the alpine lake La Caldera in the Penibetic mountains (Sierra Nevada). Verh. Internat. Verein. Limnol., 19:1133-1139.

MARTINEZ, R. -1977- Phytoplancton species, biomas and diversity in lake La Caldera (Sierra Nevada). Acta Hydrobiol., 19(2):95-107.

MARTINEZ, R. -1980- Seasonal variation of phytoplankton biomas and photosinthesis in the high mountain lake La Caldera. Developm. Hydrobiol., 3:111-148.

MARTINEZ, R. -1981- Nota sobre la migración vertical diaria de Rhodomas minuta var. nannoplanktonica en la laguna de La Caldera. Bol. R. Soc. Esp. Hist. Nat., 78:217-221.

MESSIKOMMER, E. -1942- Beiträge zur Kenntnis der Algenflora und Algenvegetation des Hochgebirges und Davos. Beitr. Geobot. Lande-saufnahme Schweiz, 24:1-451.

MESSIKOMMER, E. -1957- Algen aus den Otztaler Alpen. Archiv für Hydrobiologia, 53(4):552-561.

MONTIEL FRADES, J.C. -1947- Contribución al conocimiento de las algas de agua dulce del Pirineo aragonés. Anales Jard. Bot. Madrid, 8:259-263.

PASCHER, A. -1914- Uber Symbiosen von Spaltpilzen und Flagellaten mit Blaualgen. Bereich. Deutsch. Bot. Gesellschaft, 26:300-370.

PATRICK, R \& REIMER, O.W. -1966- The diatoms of United States (1) $688 \mathrm{pp}$. Monograph of the Academy of Natural Sciences of Philadelphia. Philadelphia.

PRESCOTT, G.W. \& VINYARD, W.C. -1965- Ecology of Alaskan fresh-water algae V. Limnology and flora of Malikpuk lake. Transactions of American Microscopical Society, 84:427-479.

PRESCOTT, G.W., CROADSLE, H.T., VINYARD, W.C. \& BICUDO, C.E. -1981- A synopsis of north american desmids. Part II. Desmidiaceae: Placodermae. Section 3. $385 \mathrm{pp}$. University of Nebraska Press. Lincoln.

SANCHEZ CASTILLO, P.M. -1984- Estudio ficológico de la vegetación reófila de Sierra Nevada (Granada). Limnetica, 1(1):136-140.

SANCHEZ CASTILLO, P.M. -1987a- Un nuevo taxon del género Chromulina: C. nevadensis P.M. Sánchez sp. nov. Cryptogamie-algologie, 8(4):235-240.

SANCHEZ CASTILLO, P.M. -1987b- Estudio del ciclo biológico de Korshicoviella gracilipes (Lambert) Silva (Chlorococcales, Chlorophyta). Phycologia, (en prensa).

SKUJA, H. -1964- Grunzüge der algenflora und algenvegetation der Fjeldgengenden um Abisko in Schwedisch-Lappland. Nova Acta Regiae Soc. Sci. Upsal., 18:1-465.

VILASECA, J.M. -1978- Fitoplancton de los lagos pirenaicos. 102 pp. Memoria de Licenciatura. Universidad de Barcelona. Barcelona.

WILLEN, T. -1960- Phytoplankton algae from three spanish lakes. Bot. Tidssk., 54(4):533-549. 
WUTHRICH, M. -1975- Contribution à la connaisance de la flore algologique du Park National Suisse. Les Diatomées. Commission de la Societé Helvetique des Sciences Naturelles pour les études scientifiques au Parc National, 14:273-369.

(Aceptado para su publicación el 4 de noviembre de 1987)

Dirección del autor: Departamento de Biologia Vegetal. Facultad de Ciencias. Universidad de Granada. 18001 Granada. 